\title{
Status and consolidated list of threatened medicinal plants of India
}

\author{
R. Gowthami (1) - Neelam Sharma (1) - Ruchira Pandey (1) Anuradha Agrawal $(\mathbb{D}$
}

Received: 7 September 2020/ Accepted: 29 April 2021/Published online: 25 May 2021

(C) The Author(s), under exclusive licence to Springer Nature B.V. 2021

\begin{abstract}
A wide array of medicinal plants in India, primarily used by locals for health care, have found wide acceptance and adoption globally (either directly or processed) due to distinct advantages of good results, low or no side-effects and ease of access to general public. Indigenous and traditional systems of medicine in practice since historical times have shown potential (direct or indirect as immune-boosters) against many dreaded ailments including the recent global pandemic of COVID-19. With prediction of sixth mass extinction, there is worldwide concern as majority of these plants, collected from natural stands, are also facing threat of extinction. Since 1990s concerted efforts have been directed towards assessment of threat status, the basic requirement for prioritizing conservation activity to various species of plants and animals. In literature there is staggered information regarding list of threatened plants,
\end{abstract}

R. Gowthami · N. Sharma $(\varangle) \cdot$ R. Pandey · A. Agrawal Tissue Culture and Cryopreservation Unit, ICAR-National Bureau of Plant Genetic Resources (NBPGR), Pusa Campus, New Delhi 110012, India e-mail: neelam.sharma1@icar.gov.in; sharma.nee1.59@gmail.com

R. Gowthami

e-mail: gowthami.r@icar.gov.in

R. Pandey

e-mail: ruchira.pandey@icar.gov.in

A. Agrawal

e-mail: anuradha.agrawal@icar.gov.in including medicinal plants of India, compiled at either state level or national or international level. Analysis of these publications led to collation of a consolidated list of 84 species and the same is presented here. A brief account of conservation efforts in India at national level and supportive policy framework is also included. This compilation is aimed to serve as a comprehensive reference especially for beginners, researchers, conservationists, foresters, pharmaceutical professionals as well as policy makers.

Keywords Conservation · CAMP · IUCN · Medicinal plants $\cdot$ Policy framework . Threat assessment

\section{Introduction}

Biodiversity conservation is a demanding task that includes scientific, social, and political challenges (Pelletier et al. 2018). Given the enormous diversity of species and limited resources available to undertake these conservation programs, prioritization of species for conservation, assessment of threat status and the most suitable strategy to be adopted become the basic pre-requisites. To serve this objective, the International Union for Conservation of Nature (IUCN), a membership union composed of both government and civil society organizations, was established. An IUCN 
'Red List of Threatened Species' was developed, which provides extinction risk and species distribution (Pollock et al. 2003; Brooks et al. 2004). As per IUCN classification, species are assigned a ranked threat category, such as extinct (EX), extinct in wild (EW), critically endangered (CR), endangered (EN), vulnerable (VU), near threatened (NT), least concern (LC)

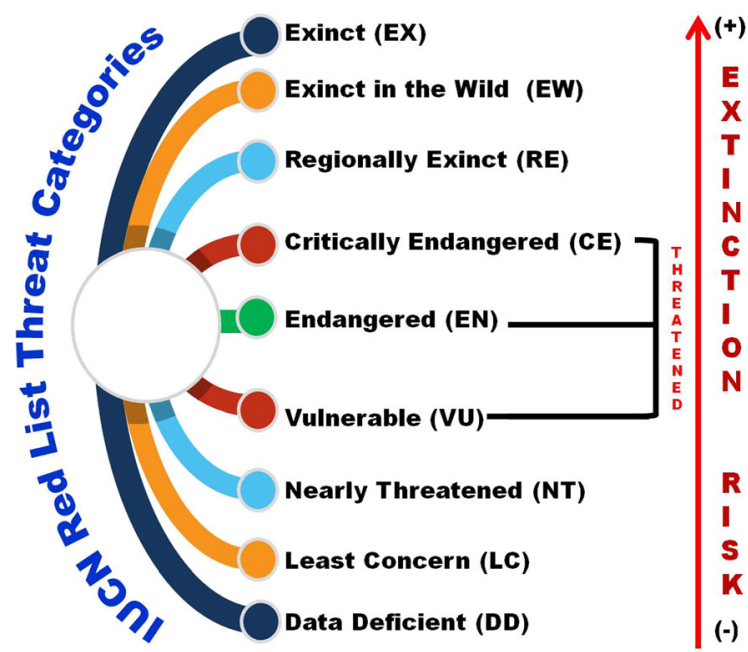

Fig. 1 IUCN Red List threat categories and their risk level and data deficient (DD) (Fig. 1) through assessment against quantitative criteria based on indicators of extinction risk (Collen et al. 2016; Hammer and Khoshbakht 2005). As the threat categories itself are basic keys to prioritize conservation this list is the globally accepted method of assessing species extinction risk (Collar 1996). Countries around the world are adapting these criteria to prioritize species for conservation, to determine the conservation approaches and to frame regulatory and policy decisions for conservation, and multilateral agreements (Maxted et al. 1997; Rodrigues et al. 2006). Figure 2 depicts the estimated number of threatened species (till date) in Kingdom Animalia, Plantae, Chromista and Fungi, globally and in India as per the IUCN assessment (IUCN 2020). Because of ever increasing human population and anthropogenic activities, species extinction rate has increased to hundred or thousandfold compared to background extinctions, bringing to forefront the 'sixth mass extinction' crisis (Shivanna 2020).

India has $2.4 \%$ of world's area with $8 \%$ of global biodiversity and is one of the 12 mega diversity countries of the world (Myers et al. 2000; Bapat et al. 2008). Among the world's 35 global biodiversity

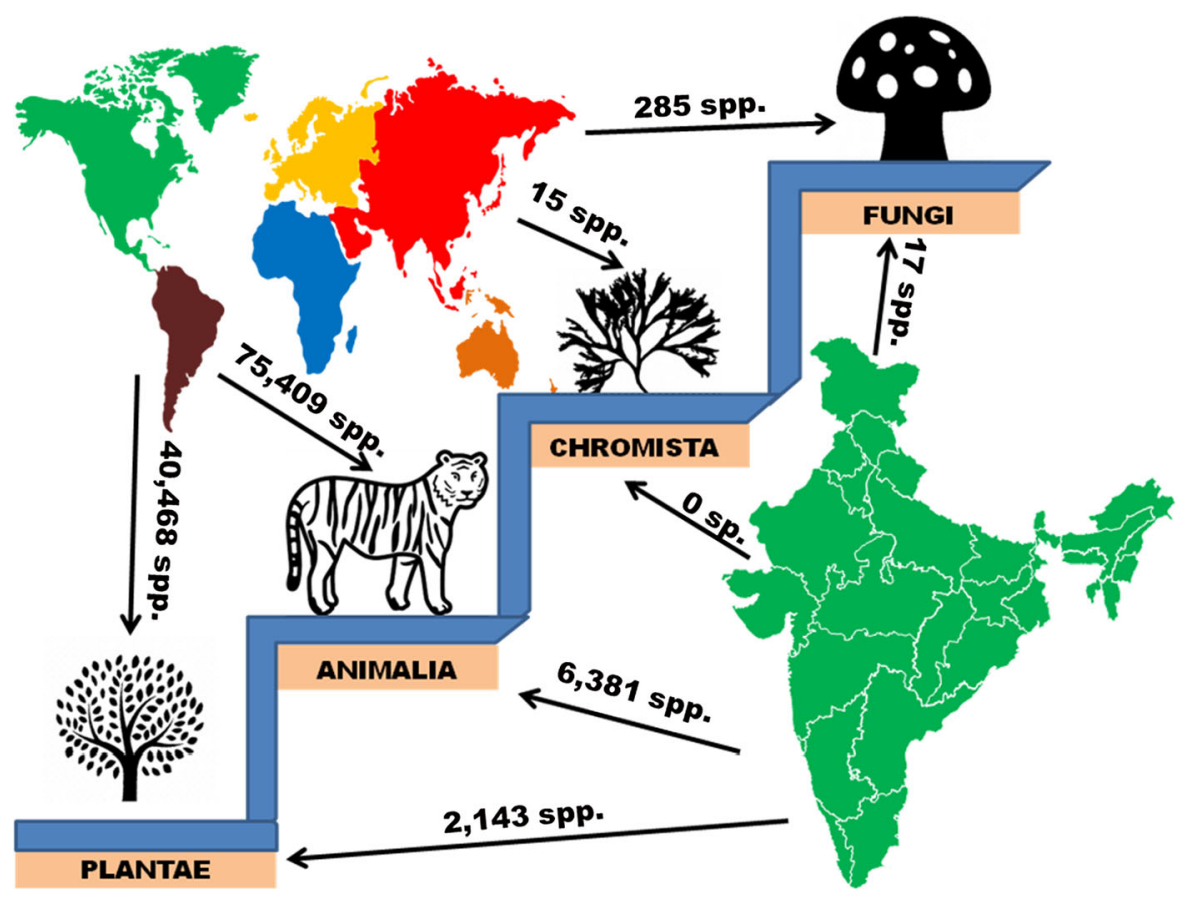

Fig. 2 Total number of threatened species of Kingdom Plantae, Animalia, Chromista and Fungi at global and national level as per IUCN estimation. Numerical values mentioned on the arrows indicate the number of threatened species (Source: IUCN 2020) 


\section{(a)}

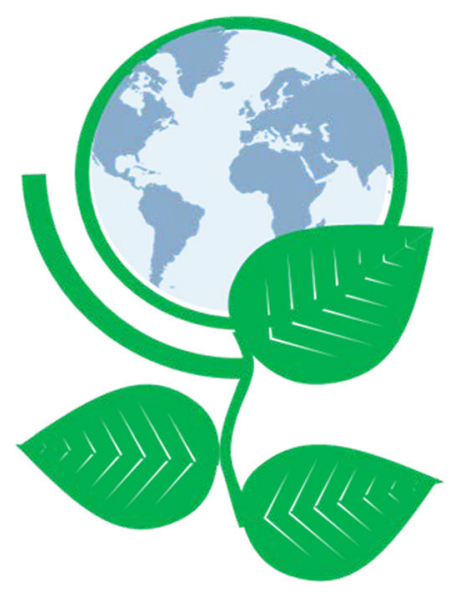

(b)

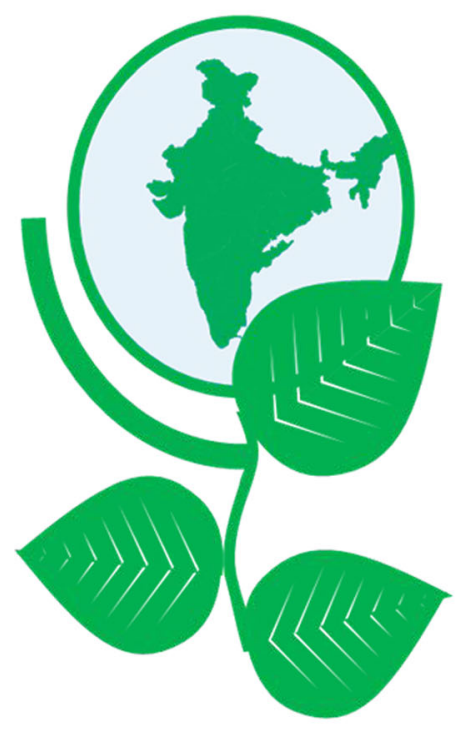

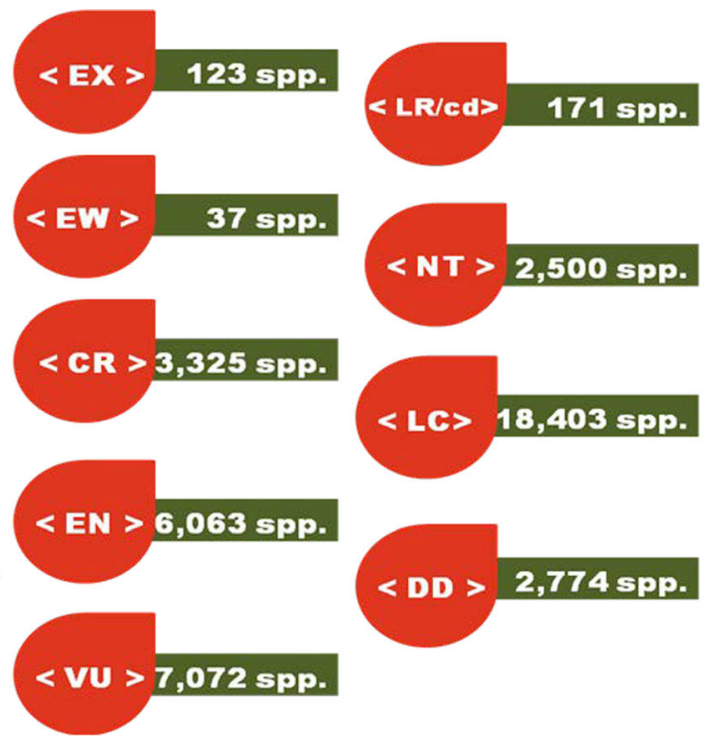

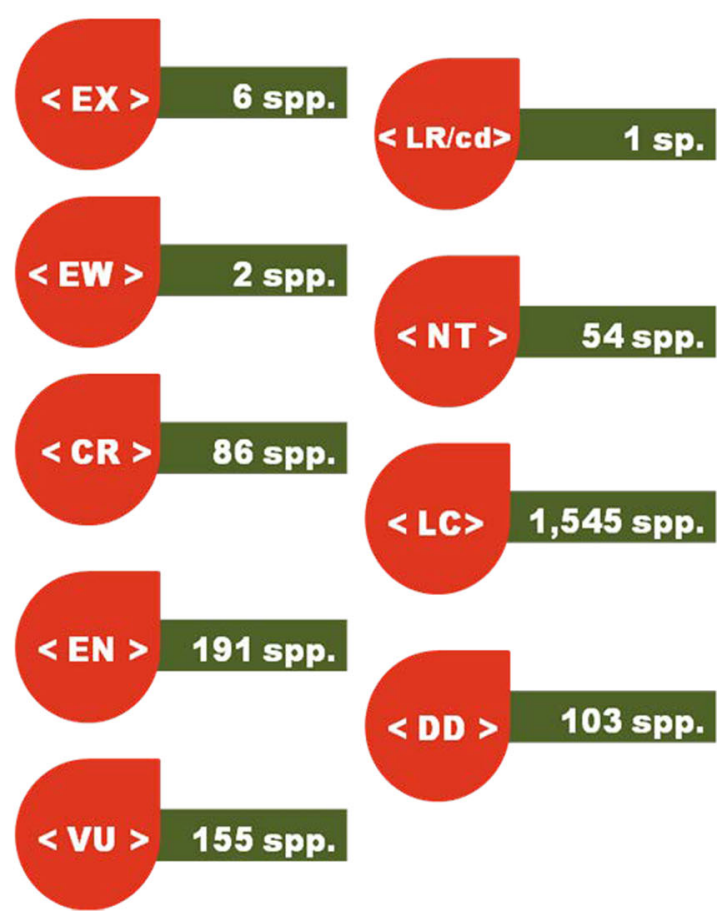

Fig. 3 Number of plant species threatened at (a) global (b) national level as per IUCN estimation (EX: Extinct; EWExtinct in the Wild; CR-Critically Endangered; EN:

hotspots $(\mathrm{GBH})$, four (Himalayas, Indo-Burma, Sundaland and Western Ghats) fall in the Indian geopolitical territory (Jaisankar et al. 2018). This richness of phyto-diversity in India is owing to existence of 15 agro-climatic zones with varied ecological habitats. India has $\sim 10.45 \%$ of global floral diversity. More
Endangered; VU: Vulnerable; LR/cd: Lower Risk-Conservation Dependent; NT: Near threatened; LC: Least concern; DD; Data deficient (Source: IUCN 2020)

than $50 \%$ of the world's plant species are endemic to $35 \mathrm{GBH}$, which once covered $15.9 \%$ of the earth's land surface and are now reduced to $2.3 \%$ (Mittermeier et al. 2011). These hotspots harbor a large number of endemic species, which are facing an increasing threat of extinction (Hazarika et al. 2016). 
Table 1 Updated list of medicinal plants under the category (medicine: plants and animals) in different threat categories as per IUCN ( Source: IUCN 2020)

Critically Endangered (CR)

Aconitum chasmanthum Stapf ex Holmes, Aquilaria malaccensis Lam. = A. agalocha Roxb.ex DC., Ceropegia odorata Nimmo ex J. Graham, Chlorophytum borivilianum Sant., Commiphora wightii (Arn.) Bhandari, Gentiana kurroo Royle., Gymnocladus assamicus Kanjilal, Lilium polyphyllum Don., Nardostachys jatamansi DC., Paphiopedilum druryi (Bedd.) Stein., Saussurea costus (Falc.) Lipsch., Tribulus rajasthanensis Bhandari \& Sharma, Utleria salicifolia Bedd., Valeriana leschenaultia DC.

Endangered (EN)

Aconitum heterophyllum Wall., Angelica glauca Edgew., Cinnamomum wightii C.F.W.Meissn, Coffea arabica L., Commiphora stocksiana Engl., Coptis teeta Wall., Curcuma caulina J. Graham, Cycas beddomei Dyer., Cycas circinalis L., Decalepis hamiltonii Wight \& Arn., Dysoxylum malabaricum Bedd., Gymnema khandalense Santapau, Humboldtia vahliana Wight, Illicium griffithii Hook. F. \& Th., Iphigenia stellata Blatter, Lamprachaenium microcephalum Benth., Nepenthes khasiana Hook.f., Pimpinella tirupatiensis Bal. \& Sub., Piper barberi Gamble., Pterocarpus indicus Willd., Shorea tumbuggaia Roxb., Syzygium alternifolium (Wt.) Wall., Taxus contorta Griff., Taxus wallichiana Zucc.

Vulnerable (VU)

Aconitum violaceum Jacq. EX Stapf, Anacyclus pyrethrum (L.) Lag., Boesenbergia siphonantha (King ex Baker) M.Sabu, Prasanthk. \& Škornick., Boswellia ovalifoliolata Bal \& Henry., Calophyllum apetalum Willd., Cayratia pedata Juss. ex Gagnepain, Cephalotaxus mannii Hook. f., Cinnamomum macrocarpum Hook., Cinnamomum sulphuratum Nees., Curcuma pseudomontana J. Graham, Cycas nathorstii J. Schust., Diospyros candolleana Wight, Diospyros paniculata Dalz.,

Dipterocarpus alatus Roxb. \& G. Don, Dipterocarpus costatus Gaertn. f., Dipterocarpus gracilis Blume, Etlingera fenzlii (Kurz) Škorničk. \& M.Sabu, Garcinia indica (Dup.) Choisy, Hopea odorata Roxb., Hydnocarpus pentandrus (Buch.-Ham.) Oken, Ilex embelioides Hook. f., Jatropha nana Dalzell \& A. Gibson, Magnolia nilagirica (Zenker) Figlar, Malaxis muscifera (Lindley) Kuntze, Myristica dactyloides Gaertn., Nilgirianthus ciliates (Nees) Bremek., Ochreinauclea missionis (Wall.ex G.Don) Ridsdale, Phyllanthus indofischeri Bennet., Piper pedicellatum C. DC., Rhynchosia heynei Wight \& Arn. = Rhynchosia coodoorensis Bedd., Salacia oblonga Wall., Santalum album L., Terminalia pallida Brandis, Vanda spathulata (L.) Spreng.

Near Threatened (NT)

Albizia thompsonii Brandis, Allium roylei Stearn, Cupressus cashmeriana Royle ex Carriere, Dioscorea hamiltonii Hook. f.,

Dipterocarpus tuberculatus Gaertn. f., Mangifera andamanica King, Pterocarpus marsupium Roxb., Pterocarpus santalinus L.f.

Least Concern (LC)

Abelmoschus crinitus Wall., Abelmoschus ficulneus (L.) Wight \& Arn. ex Wight, Acacia aneura F. Muell ex Benth., Acacia auriculiformis A. Cunn. ex Benth., Acacia nilotica (L.) Willd. ex Delile, Acanthus ebracteatus Vahl, Acanthus ilicifolius L., Acanthus volubilis Wall., Acer caesium (Reinw. Ex Blume) Kosterm, Acer laevigatum Wall., Acer pseudoplatanus L., Acmella paniculata (Wall. ex DC.) R.K.Jansen, Acorus calamus L., Acorus gramineus Aiton, Adenosma indianum (Lour.) Merr., Adenostemma viscosum J.R.Forst. \& G. Forst., Adiantum capillus-veneris L., Aegiceras corniculatum (L.) Blanco, Aeschynomene indica L., Alangium salviifolium (L. f.) Wangerin, Alchornea rugosa (Lour.) Müll.Arg., Alisma plantagoaquatica L., Alnus nepalensis D.Don, Alnus nitida (Spach) Endl., Alocasia fornicate (Roxb.) Schott, Alocasia odora (Lindl.) K.Koch, Alpinia nigra (Gaertn.) Burtt, Alternanthera sessilis (L.) R.Br. ex DC., Alysicarpus bupleurifolius (L.) DC., Ammannia auriculata Willd., Ammannia baccifera L., Amorphophallus paeoniifolius (Dennst.) Nicolson, Andira fraxinifolia Benth., Aphanamixis polystachya (Wall.) R.Parker, Archidendron ellipticum (Blanco) I.C.Nielsen, Artocarpus hirsutus Lam., Arundo donax L., Astragalus tribuloides Delile, Avicennia marina (Forssk.) Vierh., Azadirachta indica A.Juss., Azolla pinnata R. Br., Bacopa monnieri (L.) Wettst., Baphia nitida Lodd., Barringtonia acutangula L. Gaertn., Barringtonia racemosa (L.) Spreng., Bauhinia acuminata L., Bauhinia purpurea L., Beilschmiedia roxburghiana Nees., Berberis aristata DC., Betula alnoides Buch.-Ham. ex D.Don, Betula utilis D.Don, Biancaea decapetala (Roth) Alston = Caesalpinia decapetala (Roth) Alston, Bidens cernua L., Bidens tripartita L., Bischofia javanica Blume = Bischofia javanica var. toui (Decne.) Müll.Arg., Boesenbergia rotunda (L.) Mansf. = Boesenbergia pandurata (Roxb.) Schltr., Bolboschoenus maritimus (L.) Palla, Brachiaria mutica (Forssk.) Stapf, Brachiaria reptans (L.) C.A.Gardner \& C.E.Hubb, Bridelia stipularis (L.) Blume = Bridelia scandens (Roxb.) Willd., Caesulia axillaris Roxb., Cajanus scarabaeoides (L.) Benth. = Atylosia scarabaeoides (L.) Benth., Calophyllum inophyllum L., Camellia kissii Wall., Cananga odorata (Lam.) Hook.f. \& Thomson, Capparis spinosa L., Carex filicina Nees., Carex phacota Spreng., Caryota urens L., Cassia javanica L., Casuarina equisetifolia L., Catabrosa aquatica (L.) P.Beauv., Celtis australis L., Cenchrus hordeoides (Lam.) Morrone, Centella asiatica (L.) Urb. = Hydrocotyle asiatica L., Centipeda minima (L.) A.Braun \& Asch., Centrostachys aquatic (R.Br.) Moq., Cerasus cerasoides (Buch.-Ham. ex D.Don) S.Ya.Sokolov = Prunus cerasoides Buch.-Ham. ex D.Don., Ceratopteris thalictroides (L.) Brongn., Chamaecrista absus (L.) H.S.Irwin \& Barneby = Cassia absus L., Chlorophytum tuberosum (Roxb.) Baker, Christia vespertilionis (L.f.) Bakh.f., Chukrasia tabularis A.Juss., Cinnamomum tamala (Buch.-Ham.) T.Nees \& Eberm, Colocasia esculenta (L.) Schott, Colvillea racemosa Bojer, Commelina benghalensis L., Commelina clavata C.B.Clarke, Commelina diffusa Burm.f., Commelina erecta L., Commelina imberbis Ehrenb. ex Hassk., Cordia dichotoma (Ruiz \& Pav.) Gürke = Cordia bifurcata Roem. \& Schult., Cotinus coggygria Scop., Cressa cretica L., Crinum viviparum (Lam.) R.Ansari \& V.J.Nair, Crotalaria albida Roth, Crotalaria 
Table 1 continued

Critically Endangered (CR)

Aconitum chasmanthum Stapf ex Holmes, Aquilaria malaccensis Lam. = A. agalocha Roxb.ex DC., Ceropegia odorata Nimmo ex J. Graham, Chlorophytum borivilianum Sant., Commiphora wightii (Arn.) Bhandari, Gentiana kurroo Royle., Gymnocladus assamicus Kanjilal, Lilium polyphyllum Don., Nardostachys jatamansi DC., Paphiopedilum druryi (Bedd.) Stein., Saussurea costus (Falc.) Lipsch., Tribulus rajasthanensis Bhandari \& Sharma, Utleria salicifolia Bedd., Valeriana leschenaultia DC. assamica Benth. = Crotalaria burmannii DC., Crotalaria micans Link = Crotalaria dombeyana DC., Crotalaria paniculata Willd., Crotalaria quinquefolia L., Croton argyratus Blume, Cryptocoryne ciliate (Roxb.) Fisch. ex Wydler, Cryptocoryne retrospiralis (Roxb.) Kunth, Curanga amara Juss. = Picria fel-terrae Lour., Curcuma haritha Mangaly \& M.Sabu, Curcuma inodora Blatt., Cyanotis arcotensis R.S.Rao, Cyanotis axillaris (L.) Schult. F. = Cyanotis axillaris (L.) D.Don ex Sweet., Cyathocline purpurea (Buch.-Ham. ex D.Don) Kuntze., Cynometra ramiflora L., Cyperus alopecuroides Rottb., Cyperus articulatus L., Cyperus cephalotes Vahl., Cyperus compressus L., Cyperus cyperoides (L.) Kuntze, Cyperus difformis L., Cyperus diffuses Vahl, Cyperus distans L.f., Cyperus dives Delile, Cyperus esculentus L., Cyperus exaltatus Retz., Cyperus haspan L., Cyperus iria L., Cyperus laevigatus L., Cyperus platyphyllus Roem. \& Schult., Cyperus pumilus L. = Pycreus pumilus (L.) Nees., Cyperus stoloniferus Retz., Cyperus tuberosus Rottb. = C. rotundus L., Delonix elata (L.) Gamble = Poinciana elata L., Desmodium adscendens (Sw.) DC., Desmodium barbatum (L.) Benth., Desmodium elegans DC., Desmodium microphyllum (Thunb.) DC.. = Leptodesmia microphylla (Thunb.) H. Ohashi \& K. Ohashi (IPNI), Dichrostachys cinerea (L.) Wight \& Arn, Dicranopteris linearis (Burm. f.) Underw., Drosera burmanni Vahl., Drosera indica L., Drosera peltata Thunb., Echinochloa colona (L.) Link, Echinochloapicta (K.D.Koenig) P.W.Michael, Eclipta prostrata Kehraj., Eclipta alba (L.) Hassk., Elaeagnus rhamnoides (L.) A.Nelson. = Rhamnoides hippophae Moench., Eleocharis acicularis (L.) Roem. \& Schult., Eleocharis geniculata (L.) Roem. \& Schult., Eleusine indica (L.) Gaertn, Engelhardtia roxburghiana Lindl., Enhalus acoroides (L.f.) Royle, Enydra fluctuans DC., Epaltes divaricata (L.) Cass., Ephedra foliata Boiss. ex C.A.Mey., Ephedra intermedia Schrenk \& C.A.Mey., Ephedra kardangensis P.Sharma \& P.L.Uniyal, Ephedra major Host, Epilobium hirsutum L., Epilobium latifolium L., Erythrina variegata L., Ethulia conyzoides L.f., Euryale ferox Salisb., Excoecaria agallocha L., Fimbristylis dichotoma (L.) Vahl, Floscopa scandens Lour., Fraxinus floribunda Wall., Fuirena umbellata Rottb., Gloriosa superba L., Gmelina arborea Roxb., Gmelina asiatica L., Gmelina elliptica Sm. = G. villosa Roxb., Gnetum gnemon L., Gnetum montanum Markgr., Gnetum ula Brongn. = G. pyrifolium Miq. Ex Parl., Gordonia obtusa Wall. ex Wight, Grangea maderaspatana (L.) Poir., Grewia asiatica L., Grewia villosa Willd., Harpullia arborea (Blanco) Radlk., Helicia nilagirica Bedd., Heliciopsis terminalis (Kurz) Sleumer = Helicia terminalis Kurz, Heliotropium ovalifolium Forssk. = Euploca ovalifolia (Forssk.) Diane \& Hilger, Hemarthria compressa (L.f.) R.Br., Hemisteptia lyrata Fisch. \& C.A.Mey., Heritiera littoralis Aiton, Hibiscus tiliaceus L., Homonoia retusa (Graham ex Wight) Müll.Arg., Homonoia riparia Lour., Hoppea dichotoma Willd, Hydrilla verticillata (L.f.) Royle, Hydrocotyle sibthorpioides Lam., Hydrolea zeylanica (L.) Vahl, Hygrophila auriculata (Schumach.) Heine., Hygrophila pinnatifida (Dalzell) Sreem., Hygrophila polysperma (Roxb.) T.Anderson, Hygrophila salicifolia (Vahl) Nees., Hygrophila schulli M.R.Almeida \& S.M.Almeida = H. auriculata (Schumach.) Heine., Indigofera linifolia (L. f.) Retz., Indigofera oblongifolia Forssk., Indigofera trita L. f., Ipomoea aquatica Forssk., Ipomoea cairica (L.) Sweet, Ipomoea coptica (L.) Roth ex Roem. \& Schult., Iris pseudacorus L. = Limnirion pseudacorus (L.) Opiz, Ixora grandifolia Zoll. \& Moritzi, Juglans regia L., Juncus decipiens (Buchenau) Nakai, Juncus effuses L., Juniperus communis L., Knema globularia (Lam.) Warb., Kyllinga brevifolia Rottb., Kyllinga bulbosa P.Beauv., Kyllinga nemoralis (J.R.Forst. \& G.Forst.) Dandy ex Hutch. \& Dalziel., Lagenandra ovate (L.) Thwaites, Lagenandra toxicaria Dalzell, Lasia spinosa (L.) Thwaites, Lemna minor L., Leptochilus decurrens Blume, Lespedeza cuneata (Dum.Cours.) G.Don, Limnophila aromatica (Lam.) Merr., Limnophila indica (L.) Druce, Limnophila polystachya Benth., Limnophila repens (Benth.) Benth., Limnophila rugosa (Roth) Merr., Limnophyton obtusifolium (L.) Miq., Lindernia anagallis (Burm.f.) Pennell, Lindernia antipoda (L.) Alston, Lindernia ciliate (Colsm.) Pennell, Lindernia crustacea (L.) F.Muell. = Torenia crustacean (L.) Cham. \& Schltdl., Lindernia oppositifolia (L.) Mukerjee, Lindernia procumbent (Krock.) Philcox, Lindernia pusilla (Willd.) Bold., Lindernia ruellioides (Colsm.) Pennell, Liquidambar excelsa (Noronha) Oken = Altingia excelsa Noronha, Lobelia heyneana Schult., Ludwigia hyssopifolia (G.Don) Exell, Ludwigia octovalvis (Jacq.) P.H.Raven., Ludwigia perennis L., Lygodium microphyllum Link. = L. japonicum (Thunb.) Sw., Magnolia baillonii Pierre, Magnolia champaca (L.) Baill. ex Pierre = Michelia champaca L., Magnolia oblonga (Wall. ex Hook.f. \& Thomson) Figlar, Malus baccata (L.) Borkh., Marsilea minuta L., Marsilea quadrifolia L., Mecopus nidulans Benn., Medicago sativa L., Meistera masticatorum (Thwaites) Skornick.\& M.. F. Newman, Melia azedarach L., Melicope lunu-ankenda (Gaertn.) T.G. Hartley, Mentha arvensis L., Menyanthes trifoliate L., Merremia gangetica Cufod., Mimusops elengi L., Monochoria hastate (L.) Solms, Monochoria korsakowii Regel \& Maack, Monochoria vaginalis (Burm. f.) C. Presl, Mucuna bracteata DC., Musa acuminate Colla, Musa balbisiana Colla, Myriophyllum indicum Willd., Myriophyllum oliganthum (Wight \& Arn.) F. Muell., Myriophyllum tuberculatum Roxb., Myriophyllum verticillatum L., Nageia wallichiana (C.Presl) Kuntze, Nasturtium officinale R.Br., Neottia acuminate Schltr., Nephrolepis undulate (Afzel. ex Sw.) J. Sm., Nerium oleander L., Nymphaea alba L., Nymphaea nouchali Burm.f., Nymphaea pubescens Willd., Nymphaea tetragona Georgi, Nymphoides hydrophylla (Lour.) Kuntze, Oenanthe javanica (Blume) DC., Oldenlandia diffusa (Willd.) Roxb., Osmunda hugeliana C.Presl, Osmunda regalis L., Ottelia alismoides (L.) Pers., Oxystelma esculentum (L. f.) Sm., Palaquium obovatum (Griff.) Engl., Parkia timoriana (DC.) Merr., Paspalidium flavidum (Retz.) A.Camus, Paspalum conjugatum P.J.Bergius., Paspalum distichum L., Paspalum scrobiculatum L., Peltophorumafricanum Sond., Persicaria amphibian (L.) Delarbre, Persicaria barbata (L.) H. Hara, Persicaria glabrum (Willd.) M.Gómez, Persicaria lapathifolia (L.) 
Table 1 continued

Critically Endangered (CR)

Aconitum chasmanthum Stapf ex Holmes, Aquilaria malaccensis Lam. = A. agalocha Roxb.ex DC., Ceropegia odorata Nimmo ex J. Graham, Chlorophytum borivilianum Sant., Commiphora wightii (Arn.) Bhandari, Gentiana kurroo Royle., Gymnocladus assamicus Kanjilal, Lilium polyphyllum Don., Nardostachys jatamansi DC., Paphiopedilum druryi (Bedd.) Stein., Saussurea costus (Falc.) Lipsch., Tribulus rajasthanensis Bhandari \& Sharma, Utleria salicifolia Bedd., Valeriana leschenaultia DC. Delarbre, Persicaria strigosa (R.Br.) Nakai, Phragmites australis (Cav.) Trin. ex Steud., Phyla nodiflora (L.) Greene, Phyllodium pulchellum (L.) Desv., Pistacia khinjuk Stocks, Pistia stratiotes L., Plantago major L., Polygonum punctatum Elliott, Pometia pinnata J.R.Forst. \& G.Forst., Pongamia pinnata (L.) Pierre, Potamogeton crispus L., Potamogeton nodosus Poir., Potamogeton perfoliatus L., Potamogeton pusillus L., Potentilla supine L., Prunella vulgaris L., Psidium guajava L., Psidium guineense Sw., Pterospermum diversifolium Blume, Quercus acutissima Carruth., Quercus glauca Thunb., Ranunculus sceleratus L., Rhynchosia minima (L.) DC., Sagittaria guayanensis Kunth, Sagittaria trifolia L., Schima khasiana Dyer, Schima wallichii Choisy, Scleria lithosperma (L.) Sw., Scyphiphora hydrophylacea C.F.Gaertn., Sesbania bispinosa (Jacq.) W.Wight, Silene vulgaris (Moench) Garcke, Sirhookera lanceolata (Wight) Kuntze, Smithia sensitive Aiton, Sonneratia alba Sm., Sonneratia apetala Buch.-Ham., Spathodea campanulata P.Beauv., Spatholobus parviflorus (DC.) Kuntze, Sphaeranthus africanus L., Sphaeranthus amaranthoides Burm.f., Sphaeranthus indicus L., Sphaeranthus senegalensis DC., Spirodela polyrhiza (L.) Schleid., Tacca leontopetaloides (L.) Kuntze, Tephrosia tinctoria Pers., Tephrosia villosa (L.) Pers., Terminalia calamansanai (Blanco) Rolfe., Terminalia catappa L., Thespesia populnea (L.) Sol. ex Corrêa, Toona ciliata M.Roem., Toona sinensis (Juss.) M.Roem., Toona sureni (Blume) Merr., Trapa natans L., Trema orientale (L.) Blume, Triadica cochinchinensis Lour., Trifolium pretense L., Typha angustifolia L., Typha domingensis Pers., Typha elephantine Roxb., Typha orientalis C.Presl, Typhonium flagelliforme (Lodd.) Blume, Ulex europaeus (Lodd.) Blume, Uraria picta (Jacq.) DC., Utricularia aurea Lour., Utricularia bifida L., Vallisneria natans (Lour.) H.Hara, Vallisneria spiralis L., Vanda tessellate (Roxb.) Hook. ex G.Don, Veronica anagallis-aquatica L., Vigna angularis (Willd.) Ohwi \& H.Ohashi, Vitex glabrata R.Br., Vitex quinata (Lour.) F.N.Williams, Wedelia chinensis (Osbeck) Merr., Wurfbainia villosa (Lour.) Skornick. \& A.D.Poulsen, Xylia xylocarpa (Roxb.) Taub., Xyris complanata R.Br., Xyris indica L., Zanthoxylum armatum DC., Zeuxine strateumatica (L.) Schltr.

Data Deficient (DD)

Abrus fruticulosus Wight \& Arn., Amomum sericeum Roxb., Asparagus filicinus Buch.-Ham. ex D.Don., Coscinium fenestratum (Goetgh.) Colebr., Dioscorea wightii Hook.f., Ephedra khurikensis P.Sharma \& P.L.Uniyal, Fraxinus micrantha Lingelsh., Limnophila pulcherrima Hook.f., Millettia fruticosa (DC.) Baker, Platanus orientalis L., Zingiber zerumbet (L.) Roscoe ex Sm.

Authority and appropriate synonyms as per The Plant List (www.theplantlist.org), International Plant Names Index (IPNI; www.ipni. org) and India Biodiversity Portal (indiabiodiversity.org)

The IUCN Red List of Threatened Plants, first published in 1998, lists more than 8,000 species under threat (Walter and Gillett 1998).

On a global basis, IUCN has estimated that about $13.49 \%$ of the world's vascular plants $(3,00,000$ species), totaling about 40,468 species are under varying degrees of threat. Many plant species are becoming extinct (123 are extinct and 37 are extinct in wild) and under threatened categories CR $(3,325)$, EN $(6,063)$, VU $(7,072)$ (Fig. 3) or vulnerable to extinction due to various reasons like population loss, loss of pollinators, loss of reproduction and/or seed germination capacity, habitat destruction (both natural and human-induced), over exploitation and loss of genetic variability (IUCN, 2020; Kala 2000, 2005). In India about $11.53 \%$ of vascular plants $(18,532)$, totaling about 2,142 species are red listed. Out of these 8 are extinct, 432 species are threatened (CR, EN and VU) and nearly 54 species are grouped under near threatened (Fig. 3) (IUCN 2020).

Nature has bestowed India with an enormous wealth of medicinal plants, due to which the country is often referred as 'Medicinal Garden' or 'Botanical Garden' of the world (Ahmadullah and Nayar 1999). Of 48,655 plant species documented (including virus, bacteria, algae, fungi and lichens) 9,500 species have ethno-botanical importance and 7,500 species are in medicinal use for indigenous health practices as well as modern system of medicines (Kumar et al. 2013; Sharma and Pandey 2013). From ancient period medicinal plants have been used in all cultures as a source of medicine and its use as herbal medicine is described in numerous ancient texts. Besides being a major source of raw material for the traditional healthcare practices (Ayurveda, Siddha, Unani, Homeopathy, Naturopathy, Sowa-Rigpa and diverse 
Fig. 4 State-wise proportion of medicinal plants of India in different threat categories (except LC and DD) based on CAMP exercise (EX: Extinct; CRCritically endangered; EN: Endangered; VU: Vulnerable; NT: Near threatened) ( Source: Ved and Tandon 1998; Jadhav et al. 2001; Ved et al. 2003a, b; Ved et al. 2005; Goraya et al. 2013; Ved et al. 2016a, b)

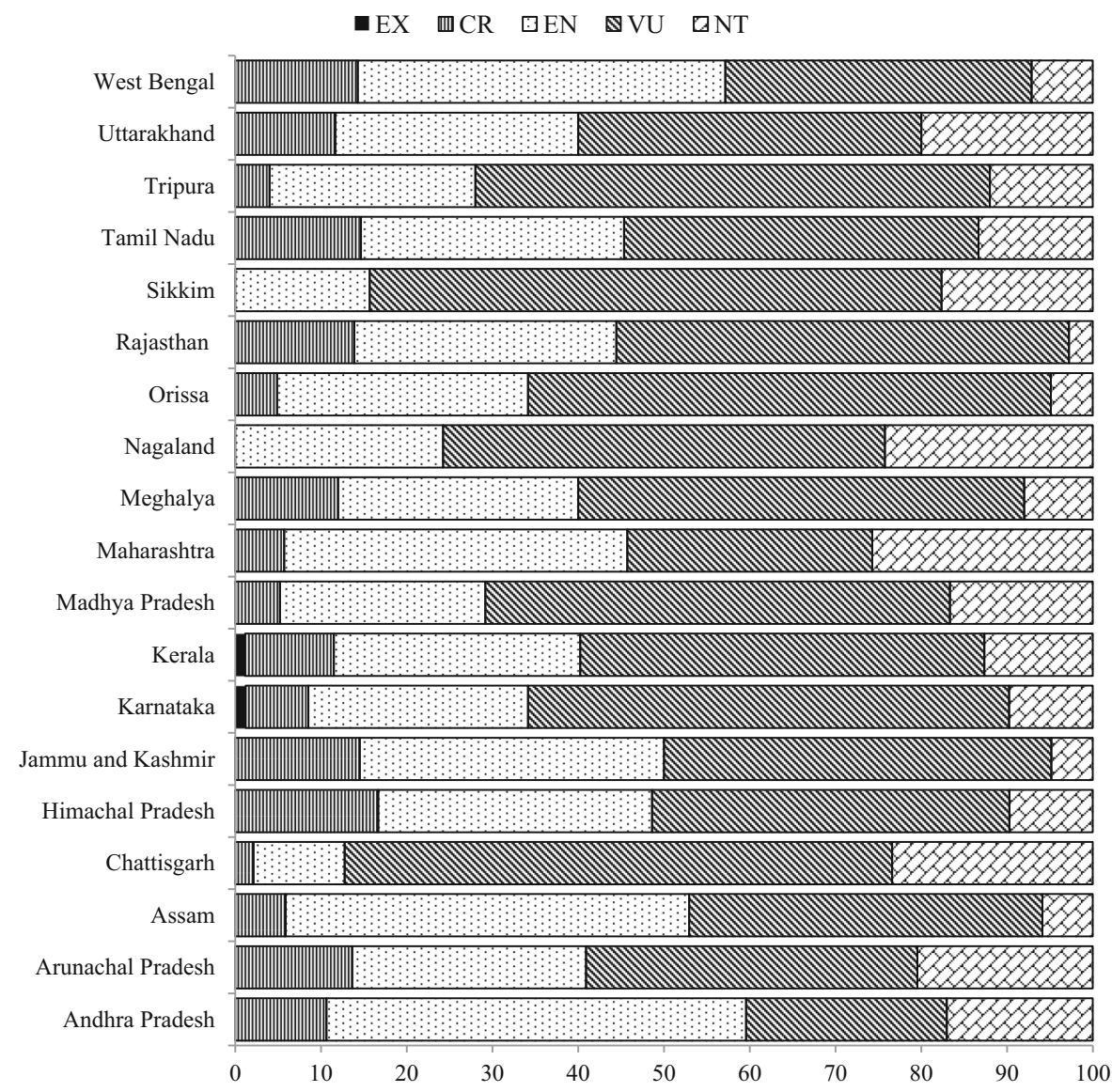

area- and community-specific folk medicine) and pharmaceutical industry, also provide livelihood to a large Indian population (Kumar et al. 2015). Indigenous and traditional systems of medicine using plants have shown potential (direct or indirect as immuneboosters) against many dreaded ailments including the recent global pandemic of COVID-19. For example Ayurveda preparations ('rasayana') with 'Ashwagandha' (Withania somnifera Dunal) can be a potential candidate for management of COVID-19, as also 'Shatavari' (Asparagus racemosus Willd.), 'Amala' (Phyllanthus emblica L. syn Embelica officinalis), 'Guduchi' [Tinospora cordifolia (Willd.) Miers], as these have immunomodulatory properties, and may have the potential to boost health and immunity to fight against SARS-CoV-2 infection (Patwardhan et al. 2020).

In India more than $90 \%$ of medicinal plants are facing threat due to excessive and unsustainable collection, utilization, overexploitation or un-skilled harvesting (Kumari et al. 2011). Based on global rates of plant species threatened with extinction, it is estimated that around 1,000 medicinal plant species may be under threat in different eco-systems across India (FRLHTENVIS 2016a). As per the IUCN Red List, a total of 457 species out of 2,143 species are listed under medicine for human and veterinary group (Table 1). Of these, 73 are threatened (CR, EN, VU), 8 (NT), 1 (DD) and 366 (LC). Although there are publications enlisting threatened plants of medicinal value at state, region, national and global levels prepared by Conservation Assessment and Management Prioritization (CAMP), Botanical Survey of India (BSI) and IUCN, etc., there is no consolidated compilation at one place, to get an accurate assessment. Therefore, in this paper an attempt has been made to review the existing information and compile an exhaustive list of threatened medicinal plants of India. This easy, one-stop-shop ready reckoner for information related to Indian threatened medicinal 

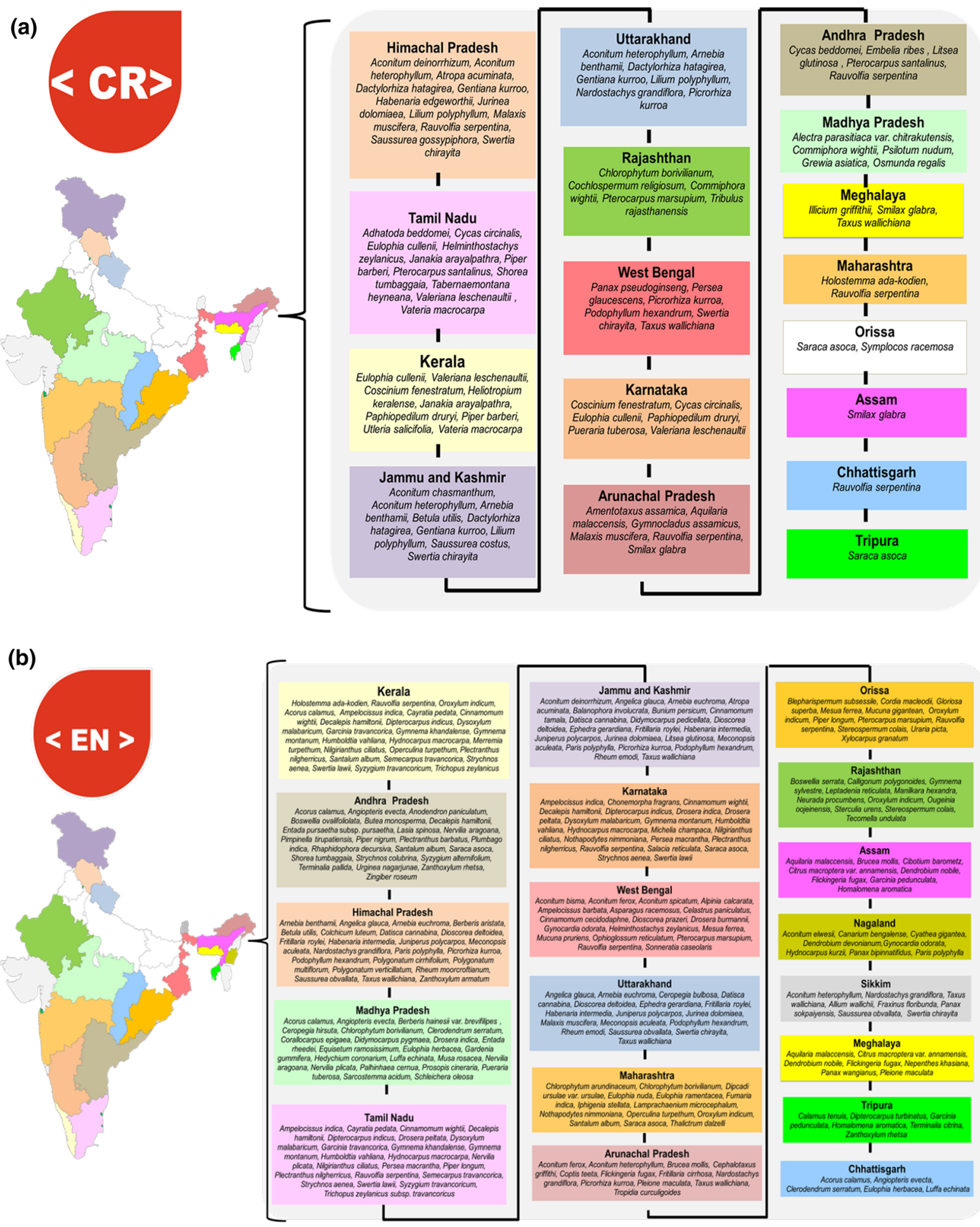

Fig. 5 State-wise list of threatened medicinal plants of India based on CAMP exercise represented from state with highest number of species to state with lowest number of species under different threat categories (a) Critically endangered (b) Endangered (c) Vulnerable. Same colour represents the state on the map. ( Source: Ved and Tandon 1998; Jadhav et al. 2001; Ved et al. 2003a, b; Ved et al. 2005; Goraya et al. 2013; Ved et al. 2016a, b) 

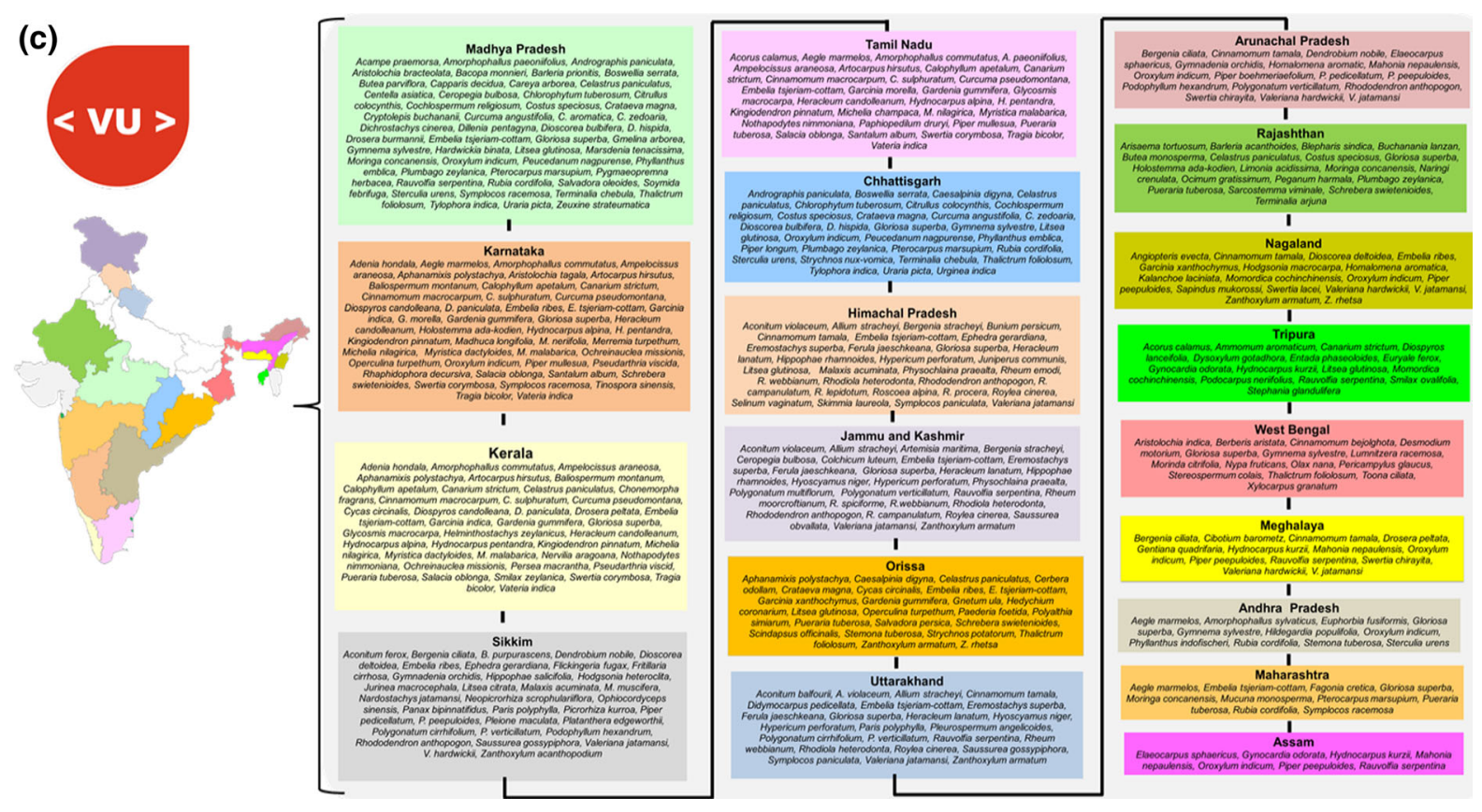

Fig. 5 continued

plant species will be beneficial especially for young researchers to strategize conservation, repatriation and use of such species. It will help not only the researchers but also policy makers in developing strategies for efficient conservation/cultivation to ensure availability of these precious resources for utilization by future generations.

\section{Threat assessment}

At national level several institutes have assessed the threat status of medicinal plants in India (Jain and Rao 1983; Nayar and Shastry 1987-1990). During 1987-1990, a total of 602 vascular plants were listed as threatened in Red Data Book of Indian Plants, which increased to 1,255 in 2003 (Rao et al. 2003), and 2,152 in 2020 (IUCN 2020). Over the past two decades, 14 CAMP workshops covering 17 states of India were conducted for rapid assessment of prioritized medicinal plant species of conservation concern in different states/regions of India by the Conservation Breeding Specialist Group (CBSG), India, in collaboration with Foundation for Revitalization of Local Health Traditions (FRLHT) (Fig. 4). CAMP workshop also uses IUCN threat categories of the respective period for assigning threat status. State-wise CAMP list of threatened medicinal plants is presented in Fig. 5a-c. We compared IUCN and CAMP list of medicinal plants and observed inconsistencies in threat status reported, and also few species missing (Table 2).

It is estimated that about 7,500 plants are used in local health traditions in most rural and tribal villages in India (Shakya 2016). Herbal treatments are the most popular form of traditional medical system (Sarker and Nahar 2007). The various codified Indian systems of medicine are complemented by a strong inheritance of non-codified and non-commercial living folk healthcare traditions. These folk healthcare traditions, carried from generation to generation and practiced by around one million folk practitioners are spread across 29 states, 7 union territories, 497 cities, 7,935 towns and 6,49,481 villages and nearly 140 million rural households in the country. The first effort to assess the demand and supply of medicinal plants of selected 162 species in the country was made by the National Medicinal Plant Board (NMPB) during 2001-02, through Centre for Research, Planning and Action (CERPA) (Goraya and Ved 2017). In 2003, export potential of medicinal plants was assessed by the FRLHT through Export Import (EXIM) Bank (Goraya 
Table 2 Consolidated list of threatened medicinal plants represented in both IUCN (India region) and CAMP exercise (India region)

\begin{tabular}{|c|c|c|}
\hline \multirow[t]{2}{*}{ Species } & \multicolumn{2}{|c|}{ Threat Status } \\
\hline & $\begin{array}{l}\text { IUCN } \\
\text { threat } \\
\text { category }\end{array}$ & $\begin{array}{l}\text { CAMP exercise threat category } \\
\text { [State (threat category and year of CAMP exercise)] }\end{array}$ \\
\hline $\begin{array}{l}\text { Aconitum chasmanthum Stapf ex } \\
\text { Holmes }\end{array}$ & $\mathrm{CR}$ & Jammu and Kashmir (CR 2003) \\
\hline Aconitum heterophyllum Wall. & EN & $\begin{array}{l}\text { Arunachal Pradesh (EN 2003), Himachal Pradesh (CR 2003, CR 2010), Jammu } \\
\text { and Kashmir (CR 2003), Sikkim (EN 2003), Uttarakhand (CR 2003) }\end{array}$ \\
\hline Aconitum violaceum Jacq. ex Stapf. & VU & $\begin{array}{l}\text { Himachal Pradesh (VU 2003, VU 2010), Jammu and Kashmir (VU 2003), } \\
\text { Uttarakhand (VU 2003) }\end{array}$ \\
\hline Acorus calamus L. & $\mathrm{LC}$ & $\begin{array}{l}\text { Andhra Pradesh (EN 2001), Chhattisgarh (EN 2003), Kerala (EN 1999), Madhya } \\
\text { Pradesh (EN 2003), Tamil Nadu (VU 1998), Tripura (VU 2016) }\end{array}$ \\
\hline $\begin{array}{l}\text { Amorphophallus paeoniifolius } \\
\text { (Dennst.) Nicolson }\end{array}$ & $\mathrm{LC}$ & Kerala (NT 1999), Madhya Pradesh (VU 2006), Tamil Nadu (VU 1998) \\
\hline Angelica glauca Edgew. & EN & $\begin{array}{l}\text { Himachal Pradesh (EN 2003, EN 2010), Jammu and Kashmir (EN 2003), } \\
\text { Uttarakhand (EN 2003) }\end{array}$ \\
\hline $\begin{array}{l}\text { Aphanamixis polystachya (Wall.) } \\
\text { R.Parker }\end{array}$ & $\mathrm{LC}$ & Karnataka (VU 1997), Kerala (VU 1997), Orissa (VU 2007) \\
\hline $\begin{array}{l}\text { Aquilaria malaccensis Lam. }=A . \\
\text { agalocha Roxb.ex DC }\end{array}$ & $\mathrm{CR}$ & $\begin{array}{l}\text { Arunachal Pradesh (CR 2003), Assam (EN 2003), Meghalaya (EN 2003), Tripura } \\
\text { (DD 2016) }\end{array}$ \\
\hline Artocarpus hirsutus Lam. & $\mathrm{LC}$ & Karnataka (VU 1999), Kerala (VU 1999), Tamil Nadu (VU 1998) \\
\hline Bacopa monnieri (L.) Wettst. & $\mathrm{LC}$ & Madhya Pradesh (VU 2006) \\
\hline Berberis aristata DC. & $\mathrm{LC}$ & Himachal Pradesh (EN 2010), Sikkim (DD 2014), West Bengal (VU 2007) \\
\hline Betula utilis D. Don & $\mathrm{LC}$ & $\begin{array}{l}\text { Himachal Pradesh (EN 2003, EN 2010), Jammu and Kashmir (CR 2003), Sikkim } \\
\text { (NT 2014), Uttarakhand (NT 2003) }\end{array}$ \\
\hline $\begin{array}{l}\text { Boswellia ovalifoliolata Bal \& } \\
\text { Henry }\end{array}$ & VU & Andhra Pradesh (EN 2001) \\
\hline Calophyllum apetalum Willd. & VU & Karnataka (VU 1999), Kerala (VU 1999), Tamil Nadu (VU 1998) \\
\hline Cayratia pedata Juss. ex Gagnepain & VU & Kerala (EN 1999), Tamil Nadu (EN 1998) \\
\hline $\begin{array}{l}\text { Centella asiatica }(\mathrm{L} .) \\
\text { Urb. = Hydrocotyle asiatica } \mathrm{L} .\end{array}$ & $\mathrm{LC}$ & Madhya Pradesh (VU 2006) \\
\hline $\begin{array}{l}\text { Chlorophytum borivilianum } \\
\text { Santapau \& R.R.Fern. }\end{array}$ & $\mathrm{CR}$ & Madhya Pradesh (EN 2006), Maharashtra (EN 2001), Rajasthan (CR 2007) \\
\hline $\begin{array}{l}\text { Chlorophytum tuberosum (Roxb.) } \\
\text { Baker }\end{array}$ & $\mathrm{LC}$ & Chhattisgarh (VU 2003), Madhya Pradesh (VU 2003) \\
\hline Cinnamoтиm macrocarpum Hook. & VU & Karnataka (VU 1999), Kerala (VU 1999), Tamil Nadu (VU 1998) \\
\hline Cinnamomum sulphuratum Nees & VU & Karnataka (VU 1999), Kerala (VU 1999), Tamil Nadu (VU 1998) \\
\hline $\begin{array}{l}\text { Cinnamomum tamala (Buch.-Ham.) } \\
\text { T.Nees \& Eberm }\end{array}$ & $\mathrm{LC}$ & $\begin{array}{l}\text { Arunachal Pradesh (VU 2003), Himachal Pradesh (VU 2003, VU 2010), Jammu } \\
\text { and Kashmir (EN 2003), Meghalaya (VU 2003), Nagaland (VU 2015), } \\
\text { Uttarakhand (VU 2003) }\end{array}$ \\
\hline $\begin{array}{l}\text { Cinnamomum wightii } \\
\text { C.F.W.Meissn }\end{array}$ & EN & Karnataka (EN 1999), Kerala (EN 1999), Tamil Nadu (EN 1998) \\
\hline $\begin{array}{l}\text { Commiphora wightii (Arn.) } \\
\text { Bhandari }\end{array}$ & $\mathrm{CR}$ & Madhya Pradesh (CR 2003), Rajasthan (CR 2007) \\
\hline Coptis teeta Wall. & EN & Arunachal Pradesh (EN 2003) \\
\hline $\begin{array}{l}\text { Coscinium fenestratum (Goetgh.) } \\
\text { Colebr. }\end{array}$ & DD & Karnataka (CR 1999), Kerala (CR 1999) \\
\hline Curcuma pseudomontana Graham & VU & Karnataka (VU 1999), Kerala (EN1999), Tamil Nadu (EN 1998) \\
\hline Cycas beddomei Dyer & EN & Andhra Pradesh (CR 2001) \\
\hline
\end{tabular}


Table 2 continued

\begin{tabular}{|c|c|c|}
\hline \multirow[t]{2}{*}{ Species } & \multicolumn{2}{|c|}{ Threat Status } \\
\hline & $\begin{array}{l}\text { IUCN } \\
\text { threat } \\
\text { category }\end{array}$ & $\begin{array}{l}\text { CAMP exercise threat category } \\
\text { [State (threat category and year of CAMP exercise)] }\end{array}$ \\
\hline Cycas circinalis $\mathrm{L}$. & EN & $\begin{array}{l}\text { Karnataka (CR 1999), Kerala (VU 1999), Orissa (VU 2007), Tamil Nadu (CR } \\
\text { 1998) }\end{array}$ \\
\hline Decalepis hamiltonii Wight \& Arn. & $\mathrm{EN}$ & $\begin{array}{l}\text { Andhra Pradesh (EN 2001), Karnataka (EN 1999), Kerala (EN 1999), Tamil } \\
\text { Nadu (EN 1998) }\end{array}$ \\
\hline Dendrobium devosianum J.J.Sm. & DD & Nagaland (EN 2015) \\
\hline $\begin{array}{l}\text { Dichrostachys cinerea Wight \& } \\
\text { Arn. }\end{array}$ & LC & Madhya Pradesh (VU 2006) \\
\hline Diospyros candolleana Wight & VU & Karnataka (VU 1999), Kerala (VU 1999) \\
\hline Diospyros paniculata Dalz. & VU & Karnataka (VU 1999), Kerala (VU 1999) \\
\hline Drosera burmanni Vahl & $\mathrm{LC}$ & Madhya Pradesh (VU 2006), West Bengal (EN 2007) \\
\hline Drosera indica $\mathrm{L}$. & $\mathrm{LC}$ & Karnataka (EN 1999), Madhya Pradesh (EN 2006) \\
\hline Drosera peltata Thunb. & $\mathrm{LC}$ & $\begin{array}{l}\text { Arunachal Pradesh (NT 2003), Karnataka (EN 1999), Kerala (VU 1999), } \\
\text { Meghalaya (VU 2003), Tamil Nadu (EN 1998) }\end{array}$ \\
\hline Dysoxylum malabaricum Bedd. & EN & Karnataka (EN 1999), Kerala (EN 1999), Tamil Nadu (EN 1998) \\
\hline Euryale ferox Salisb. & $\mathrm{LC}$ & Tripura (VU 2016) \\
\hline Fraxinus floribunda Wall. & $\mathrm{LC}$ & Sikkim (EN 2014) \\
\hline Garcinia indica (Thouars) Choisy & VU & Karnataka (VU 1999), Kerala (VU 1999), Maharashtra (NT 2001) \\
\hline Gentiana kurroo Royle & $\mathrm{CR}$ & $\begin{array}{l}\text { Himachal Pradesh (CR 2003, CR 2010), Jammu and Kashmir (CR 2003), } \\
\text { Uttarakhand (CR 2003) }\end{array}$ \\
\hline Gloriosa superb L. & $\mathrm{LC}$ & $\begin{array}{l}\text { Andhra Pradesh (VU 2001), Chhattisgarh (VU 2003), Himachal Pradesh (VU } \\
\text { 2003), Jammu and Kashmir (VU 2003), Karnataka (VU 1999), Kerala (VU } \\
\text { 1999), Madhya Pradesh (VU 2003), Maharashtra (VU 2001), Orissa (EN 2007), } \\
\text { Rajasthan (VU 2007), Uttarakhand (VU 2003), West Bengal (VU 2007) }\end{array}$ \\
\hline Gmelina arborea Roxb. & $\mathrm{LC}$ & Madhya Pradesh (VU 2006) \\
\hline Gnetum montanum Markgr. & $\mathrm{LC}$ & Tripura (DD 2016) \\
\hline $\begin{array}{l}\text { Gnetum ula Brongn. = G. } \\
\text { pyrifolium Miq. Ex Parl. }\end{array}$ & $\mathrm{LC}$ & Orissa (VU 2007) \\
\hline Grewia asiatica $\mathrm{L}$. & $\mathrm{LC}$ & Madhya Pradesh (CR 2006) \\
\hline Gymnema khandalense Santapau & EN & Kerala (EN 1999), Tamil Nadu (EN 1998) \\
\hline Gymnocladus assamicus Kanjilal & $\mathrm{CR}$ & Arunachal Pradesh (CR 2003) \\
\hline Humboldtia vahliana Wight & EN & Karnataka (EN 1999), Kerala (EN 1999), Tamil Nadu (EN 1998) \\
\hline Illicium griffithii Hook. F. \& Th. & EN & Arunachal Pradesh (NT 2003), Meghalaya (CR 2003) \\
\hline Iphigenia stellata Blatter & EN & Maharashtra (EN 2001) \\
\hline Juniperus communis L. & $\mathrm{LC}$ & Himachal Pradesh (VU 2010) \\
\hline $\begin{array}{l}\text { Lamprachaenium microcephalum } \\
\text { Benth. }\end{array}$ & EN & Maharashtra (EN 2001) \\
\hline Lasia spinosa (L.) Thwaites & $\mathrm{LC}$ & Andhra Pradesh (EN 2001) \\
\hline Lilium polyphyllum Don & $\mathrm{CR}$ & $\begin{array}{l}\text { Himachal Pradesh (CR 2003, CR 2010), Jammu and Kashmir (CR 2003), } \\
\text { Uttarakhand (CR 2003) }\end{array}$ \\
\hline Malaxis muscifera (Lindl.) Kuntze & VU & $\begin{array}{l}\text { Arunachal Pradesh (CR 2003), Himachal Pradesh (CR 2003, CR 2010), Jammu } \\
\text { and Kashmir (NT 2003), Sikkim (EN 2003, VU 2014), Uttarakhand (EN 2003) }\end{array}$ \\
\hline Myristica dactyloides Gaertn. & VU & Karnataka (VU 1999), Kerala (VU 1999) \\
\hline Nardostachys jatamansi DC. & CR & Sikkim (VU 2014) \\
\hline Nepenthes khasiana Hook.f. & $\mathrm{EN}$ & Meghalaya (EN 2003) \\
\hline
\end{tabular}


Table 2 continued

\begin{tabular}{|c|c|c|}
\hline \multirow[t]{2}{*}{ Species } & \multicolumn{2}{|c|}{ Threat Status } \\
\hline & $\begin{array}{l}\text { IUCN } \\
\text { threat } \\
\text { category }\end{array}$ & $\begin{array}{l}\text { CAMP exercise threat category } \\
\text { [State (threat category and year of CAMP exercise)] }\end{array}$ \\
\hline $\begin{array}{l}\text { Nilgirianthus ciliates (Nees) } \\
\text { Bremek }\end{array}$ & $\mathrm{VU}$ & Karnataka (EN 1999), Kerala (EN 1999), Tamil Nadu (EN 1998) \\
\hline $\begin{array}{l}\text { Ochreinauclea missionis (Wall.ex } \\
\text { G.Don) Ridsdale }\end{array}$ & VU & Karnataka (VU 1999), Kerala (VU 1999) \\
\hline Osmunda regalis $\mathrm{L}$. & $\mathrm{LC}$ & Madhya Pradesh (CR 2006) \\
\hline Paphiopedilum druryi (Bedd.) Stein & $\mathrm{CR}$ & Karnataka (CR 1999), Kerala (CR 1999), Tamil Nadu (VU 1998) \\
\hline Phyllanthus indofischeri Bennet & VU & Andhra Pradesh (VU 2001) \\
\hline Pimpinella tirupatiensis Bal. \& Sub. & EN & Andhra Pradesh (EN 2001) \\
\hline Piper barberi Gamble & EN & Kerala (CR 1999), Tamil Nadu (CR 1998) \\
\hline Piper pedicellatum C. DC. & VU & Arunachal Pradesh (VU 2003), Sikkim (VU 2003) \\
\hline Pterocarpus marsupium Roxb. & NT & $\begin{array}{l}\text { Chhattisgarh (VU 2003), Madhya Pradesh (VU 2003), Maharashtra (VU 2001), } \\
\text { Orissa (EN 2007), Rajasthan (CR 2007), West Bengal (EN 2007) }\end{array}$ \\
\hline Pterocarpus santalinus L.f. & NT & Andhra Pradesh (CR 2001), Tamil Nadu (CR 1998) \\
\hline Salacia oblonga Wall. & VU & Karnataka (VU 1999), Kerala (VU 1999) Tamil Nadu (VU 1998) \\
\hline Santalum album $\mathrm{L}$. & VU & $\begin{array}{l}\text { Andhra Pradesh (EN 2001), Karnataka (VU 1999), Kerala (EN 1999), } \\
\text { Maharashtra (EN 2001), Tamil Nadu (VU 1998) }\end{array}$ \\
\hline Saussurea costus (Falc.) Lipsch. & $\mathrm{CR}$ & Jammu and Kashmir (CR 2003) \\
\hline Spatholobus parviflorus (L.) Engl. & $\mathrm{LC}$ & Tripura (DD 2016) \\
\hline Syzygium alternifolium (Wt.) Wall. & EN & Andhra Pradesh (EN 2001) \\
\hline Tacca leontopetaloides (L.) Kuntze & $\mathrm{LC}$ & Andhra Pradesh (NT 2001), Madhya Pradesh (NT 2006) \\
\hline Taxus wallichiana Zucc. & EN & $\begin{array}{l}\text { Arunachal Pradesh (EN 2003), Himachal Pradesh (EN 2003, EN 2010), Jammu } \\
\text { and Kashmir (EN 2003), Meghalaya (CR 2003), Sikkim (EN 2003), } \\
\text { Uttarakhand (EN 2003), West Bengal (CR 2007) }\end{array}$ \\
\hline Terminalia pallida Brandis & VU & Andhra Pradesh (EN 2001) \\
\hline $\begin{array}{l}\text { Toona ciliate } \mathrm{M} . \text { Roem }=\text { Cedrela } \\
\text { toona } \text { Roxb. ex Rottler }\end{array}$ & LC & West Bengal (VU 2007) \\
\hline $\begin{array}{l}\text { Tribulus rajasthanensis Bhandari \& } \\
\text { Sharma }\end{array}$ & $\mathrm{CR}$ & Rajasthan (CR 2007) \\
\hline Uraria picta (Jacq.) DC. & $\mathrm{LC}$ & $\begin{array}{l}\text { Chhattisgarh (VU 2003), Himachal Pradesh (NE 2010), Madhya Pradesh (VU } \\
\text { 2003), Orissa (EN 2007) }\end{array}$ \\
\hline Utleria salicifolia Bedd. & $\mathrm{CR}$ & Kerala (CR 1999) \\
\hline Valeriana leschenaultia DC. & CR & Karnataka (CR1997), Kerala (CR1997), Tamil Nadu (CR1997) \\
\hline Zanthoxylum armatum DC. & $\mathrm{LC}$ & $\begin{array}{l}\text { Himachal Pradesh (EN 2003, EN 2010), Jammu and Kashmir (VU 2003), } \\
\text { Nagaland (VU 2015), Orissa (VU 2007), Uttarakhand (VU 2003) }\end{array}$ \\
\hline Zeuxine strateumatica (L.) Schltr. & $\mathrm{LC}$ & Madhya Pradesh (VU 2006) \\
\hline
\end{tabular}

EX: Extinct; CR: Critically Endangered; EN: Endangered; VU: Vulnerable; LR: Lower Risk; LR: Lower Risk; NT: Near threatened; LC: Least concern; DD: Data deficient

IUCN Red list under medicine for human and veterinary group is used

(Source: Ved and Tandon 1998; Jadhav et al. 2001; Ved et al. 2003a, b, 2016a; Ved et al. 2005; Goraya et al. 2013IUCN 2020)

Authority and appropriate synonyms as per The Plant List (www.theplantlist.org), International Plant Names Index (IPNI; www.ipni. org) and India Biodiversity Portal (indiabiodiversity.org)

and Ved 2017). Under this study, a total of 880 medicinal plant species were found traded. NMPB also assigned to the Indian Council of Forestry
Research and Education (ICFRE) with FRLHT to reassess the demand and supply of medicinal plants in the country and developed a comprehensive database 
Table 3 Threat status of 242 plant species with high commercial demand (> 100 MT/year) in India

Species Threat status

IUCN CAMP exercise threat category

threat [State (threat category and year of CAMP exercise)]

category

Abelmoschus moschatus Medik.*

Abies spectabilis (D.Don) Mirb.

Abrus precatorius $\mathrm{L}$.

Abutilon indicum (L.) Sweet

Acacia catechu (L.f.) Willd.

Acacia nilotica subsp. indica (Benth.) Brenan

Acacia senegal (L.) Willd.

Acacia seyal Delile

Acacia sinuata (Lour.) Merr.

Acalypha indica $\mathrm{L}$.

Achillea millefolium $\mathrm{L}$.

Achyranthes aspera L.

Aconitum heterophyllum Wall. ex Royle

Acorus calamus L.*

LC

Aegle marmelos (L.) Correa

Aerva lanata (L.) Juss.

Albizia amara (Roxb.)B. Boivin

Alhagi pseudalhagi (M.Bieb.) Desv. Ex B.Keller \&

Shap. = Alhagi maurorum Medik

Aloe vera (L.) Burm.f.*

Alpinia calcarata (Haw.) Roscoe*

Alpinia galanga (L.) Willd.*

Amorphophallus paeoniifolius (Dennst.) Nicolson

Anacyclus pyrethrum (L.) Lag.

Andrographis paniculata (Burm.f.) Nees

Argyreia elliptica Arn. ex Choisy

Arnebia benthamii (Wall. Ex G.Don) Johnston

Artemisia aпnиа L.*

Asparagus adscendens Roxb.

Asparagus racemosus Willd.

Atropa belladonna L.

Azadirachta indica A.Juss.*

Baccharoides anthelmintica (L.) Moench

Bacopa monnieri (L.) Wettst.

Baliospermum montanum (Willd.) Müll.Arg

= B. solanifolium (Burm.) Suresh

$\begin{array}{ll}- & - \\ - & - \\ & \\ - & - \\ - & - \\ - & - \\ - & - \\ - & - \\ - & - \\ - & - \\ - & - \\ - & -\end{array}$

EN Arunachal Pradesh (EN 2003), Himachal Pradesh (CR 2003, CR 2010), Jammu and Kashmir (CR 2003), Sikkim (EN 2003), Uttarakhand (CR 2003)

Andhra Pradesh (EN 2001), Chhattisgarh (EN 2003), Kerala (EN 1999), Madhya Pradesh (EN 2003), Tamil Nadu (VU 1998), Tripura (VU 2016)

Andhra Pradesh (VU 2001), Karnataka (VU 1999), Maharashtra (VU2001), Tamil Nadu (VU 1998)

$-$

$-$

$-$

- $\quad$ West Bengal (EN 2007)

$-$

LC Kerala (NT 1999), Madhya Pradesh (VU 2006), Tamil Nadu (VU 1998)

VU

- $\quad$ Chhattisgarh (VU 2003), Madhya Pradesh (VU 2003)

- $\quad$ -

- $\quad$ Himachal Pradesh (CR 2003, EN 2010), Jammu and Kashmir (CR 2003), Uttarakhand (CR 2003)

$-$

$-\quad-$

- $\quad$ Chhattisgarh (NT 2003), Madhya Pradesh (NT 2003), West Bengal (EN 2007)

$-$

LC -

-

LC Madhya Pradesh (VU 2006)

- $\quad$ Chhattisgarh (NT 2003), Karnataka (VU 1999), Kerala (VU 1999), Madhya Pradesh (NT 2003) 
Table 3 continued

Species

Threat status

IUCN CAMP exercise threat category

threat [State (threat category and year of CAMP exercise)]

category

Barleria prionitis L.

Bauhinia variegata $\mathrm{L}$.

Berberis aristata DC.

Berberis lycium Royle

Bergenia ciliata (How.) Sternb.

Betula utilis D. Don

Boerhavia diffusa L.

Bombax ceiba L.

Boswellia serrata Roxb. ex Colebr.

Buchanania cochinchinensis (Lour.) M.R.Almeida

Butea monosperma (Lam.) Taub.

Caesalpinia bonduc (L.) Roxb.

Calendula officinalis L.*

Capparis spinosa $\mathrm{L}$.

Cardiospermum halicacabum $\mathrm{L}$.

Cassia fistula L.

Catharanthus roseus (L.) G.Don*

Cedrus deodara (Roxb. Ex D.Don) G.Don

Celastrus paniculatus Willd.

Centella asiatica (L.) Urb. = Hydrocotyle asiatica $\mathrm{L}$.

LC

Chamaecrista absus (L.)

LC

H.S Irwin \& Barneby

Chlorophytum borivilianum Sant.*

Chlorophytum tuberosum (Roxb.) Baker

Chrysopogon zizanioides (L.) Roberty*

Cichorium intybus L.*

Cinnamomum cassia (L.) J.Presl

Cinnamomum sulphuratum Nees

Cinnamomum tamala

(Buch.-Ham.) T.Nees \& Eberm

Cinnamomum verum J.Presl *

Cissus quadrangularis L.

Citrullus colocynthis (L.) Schrad.

Clerodendrum phlomidis L.f.

CR
Madhya Pradesh (VU 2006)

$-$

LC Himachal Pradesh (EN 2010), Sikkim (DD 2014), West Bengal (VU 2007)

$-$

Arunachal Pradesh (VU 2003), Meghalaya (VU 2003), Nagaland (NT 2015), Sikkim (VU 2003)

LC Himachal Pradesh (EN 2003, EN 2010), Jammu and Kashmir (CR 2003), Sikkim (NT 2014), Uttarakhand (NT 2003)

$-$

Chhattisgarh (VU 2003), Madhya Pradesh (VU 2003), Rajasthan (EN 2007)

$-$

Andhra Pradesh (EN 2001), Rajasthan (VU 2007)

$-$

$-$

$-$

$-$

$-$

$-$

Andhra Pradesh (NT 2001), Chhattisgarh (VU 2003),

Karnataka (NT 1999), Kerala (VU 1999), Madhya Pradesh (VU 2003), Orissa (VU 2007), Rajasthan (VU 2007), Tamil Nadu (NT 1998), West Bengal (EN 2007)

Madhya Pradesh (VU 2006)

$-$

Madhya Pradesh (EN 2006), Maharashtra (EN 2001), Rajasthan (CR 2007)

LC Chhattisgarh (VU 2003), Madhya Pradesh (VU 2003)

$-\quad-$

$-$

$-$

VU Karnataka (VU 1999), Kerala (VU 1999), Tamil Nadu (VU 1998)

LC Arunachal Pradesh (VU 2003), Himachal Pradesh (VU 2003, VU 2010), Jammu and Kashmir (EN 2003), Meghalaya (VU 2003), Nagaland (VU 2015), Uttarakhand (VU 2003)

$-\quad-$

$-$

- $\quad$ Chhattisgarh (VU 2003), Madhya Pradesh (VU 2003)

$-\quad-$ 
Table 3 continued

Species Threat status

IUCN CAMP exercise threat category

threat [State (threat category and year of CAMP exercise)] category

Clerodendrum serratum $(\mathrm{L}$.$) Moon =$ Rotheca serrata (L.) Steane \& Mabb

Coleus forskohlii (Willd.) Briq.*

Commiphora wightii (Arn.) Bhandari

Convolvulus prostratus Forskk.

Coptis teeta Wall.

Crateva religiosa G.Forst.

Cullen corylifolium (L.) Medik.

Curculigo orchioides Gaertn.

Curcuma zerumbet Roxb

= Curcuma euchroma Valeton*

Cymbopogon citratus (DC.) Stapf. *

Cymbopogon flexuosus (Nees ex Steud.)W.Watson*

Cynodon dactylon (L.) Pers.

Cyperus rotundus $\mathrm{L}$.

Cyperus scariosus R.Br.

Datura metel L.

Decalepis hamiltonii Wight \& Arn.

Desmodium gangeticum (L.) DC.

Didymocarpus pedicellatus R.Br.

Dioscorea bulbifera L.

Eclipta prostrata (L.) L.

Embelia ribes Burm.f.

Embelia tsjeriam-cottam (Roem. \& Schult.) A. DC.

Ephedra gerardiana Wall. Ex Stapf

Erythrina variegata L.

Eucalyptus globolus Labill.*

Ferula assa-foetida L.

Ficus benghalensis L.

Ficus religiosa L.*

Flickingeria macraei (Lindl.) Seidenf. = Dendrobium macraei Lindl.

Fumaria indica (Hausskn.) Pugsley

Garcinia gummi-gutta (L.) Roxb.
Chhattisgarh (EN 2003), Madhya Pradesh (EN 2003)

$-$

CR Madhya Pradesh (CR 2003), Rajasthan (CR 2007)

- $\quad$ -

EN Arunachal Pradesh (EN 2003)

$-$

$-\quad-$

$-\quad-$

$-$

$\begin{array}{ll}- & - \\ - & - \\ - & - \\ - & - \\ - & - \\ - & -\end{array}$

EN Andhra Pradesh (EN 2001), Karnataka (EN 1999), Kerala (EN 1999), Tamil Nadu (EN 1998)

Himachal Pradesh (NE 2010)

Himachal Pradesh (VU 2003, NT 2010), Jammu and Kashmir (EN 2003), Uttarakhand (VU 2003)

Chhattisgarh (VU 2003), Madhya Pradesh (VU 2003)

$\mathrm{LC} \quad-$

Andhra Pradesh (CR 2001), Arunachal Pradesh (NT 2003), Karnataka (VU 1999), Kerala (NT 1999), Nagaland (VU 2015), Orissa (VU 2007), Sikkim (VU 2003)

Chhattisgarh (NT 2003), Himachal Pradesh (VU 2003), Jammu and Kashmir (VU 2003), Karnataka (VU 1999), Kerala (NT 1999), Madhya Pradesh (VU 2003), Maharashtra (VU 2001), Orissa (VU 2007), Tamil Nadu (VU 1997), Uttarakhand (VU 2003)

Himachal Pradesh (VU 2003, VU 2010), Jammu and Kashmir (EN 2003), Sikkim (VU 2014), Uttarakhand (EN 2003)

LC -

$-$

$-$

$-$

$-$

$-$

- $\quad$ Maharashtra (EN 2001)

- $\quad$ Karnataka (NT 1999), Kerala (NT 1999), Tamil Nadu (NT 1998) 
Table 3 continued

Species

Glycyrrhiza glabra L.

Gmelina arborea Roxb.

Gymnema sylvestre (Retz.) R.Br. ex Sm.

Hedychium spicatum $\mathrm{Sm}$.

Helicteres isora $\mathrm{L}$

Hemidesmus indicus (L.) R.Br. ex Schult.

Hibiscus rosa-sinensis $\mathrm{L}$.

Holarrhena pubescens Wall. ex G.Don

Holoptelea integrifolia Planch.

Homalomena aromatica (Spreng.) Schott*

Hygrophila schulli M. R. Almeida \& S.M.

Almeida = Hygrophila auriculata (Schumach.) Heine

Hyoscyamus niger L.

Indigofera tinctoria L.*

Inula racemosa Hook.f.*

Ipomoea mauritiana Jacq.

Ipomoea nil (L.) Roth

Jasminum officinale L.*

Jasminum sambac (L.) Aiton*

Juniperus communis L.

Justicia adhatoda L.*

Justicia beddomei (C.B.Clarke) Bennet *

Kaempferia galanga L.*

Lactuca sativa $\mathrm{L}$.

Lawsonia inermis L.*

Lepidium sativum L.*

Leptadenia reticulata (Retz.) Wight \& Arn.

Litsea glutinosa (Lour.) C.B.Rob.

Madhuca indica J.F.Gmel. = Madhuca longifolia var. latifolia (Roxb.) A.Chev

Threat status

IUCN CAMP exercise threat category

threat [State (threat category and year of CAMP exercise)]

category

VU

LC

Anc
Karnataka (VU 1999), Kerala (VU 1999), Maharashtra (NT 2001)
Andhra Pradesh (VU 2001), Chhattisgarh (VU 2003), Himachal Pradesh (VU 2003), Jammu and Kashmir (VU 2003), Karnataka (VU 1999), Kerala (VU 1999), Madhya Pradesh (VU 2003), Maharashtra (VU 2001), Orissa (EN 2007), Rajasthan (VU 2007), Uttarakhand (VU 2003), West Bengal (VU 2007)

-

LC Madhya Pradesh (VU 2006)

Andhra Pradesh (VU 2001), Chhattisgarh (VU 2003),

Madhya Pradesh (VU 2003), Maharashtra (NT 2001),

Rajasthan (EN 2007), West Bengal (VU 2007)

Nagaland (LC 2015), Sikkim (LC 2014)

$-$

$-$

$-$

$-$

$-$

$-$

$-$

Arunachal Pradesh (VU 2003), Assam (EN 2003), Nagaland (VU 2015), Tripura (EN 2016)

$\mathrm{LC} \quad-$

Himachal Pradesh (EN 2003, NT 2010), Jammu and Kashmir (VU 2003), Uttarakhand (VU 2003)

$-$

$-$

West Bengal (NT 2007)

$-$

$-$

$-$

LC Himachal Pradesh (VU 2010)

$-$

$-$

- $\quad$ Tripura (DD 2016)

- n -

$-$

$-\quad-$

- $\quad$ Rajasthan (EN 2007)

- $\quad$ Andhra Pradesh (CR 2001), Chhattisgarh (VU 2003), Himachal Pradesh (VU 2003, VU2010), Jammu and Kashmir (EN 2003), Madhya Pradesh (VU 2003), Orissa (VU 2007), Tripura (VU 2016), Uttarakhand (NT 2003) 
Table 3 continued

Species Threat status

IUCN CAMP exercise threat category

threat [State (threat category and year of CAMP exercise)] category

Madhuca longifolia (J.Koenig ex L.) J.F.Macbr.

Martynia annua $\mathrm{L}$.

Melaleuca leucadendra (L.) L.

Melia azedarach L.*

Mentha longifolia (L.) L.

Mesua ferrea $\mathrm{L}$.

Mimusops elengi L.

Morinda citrifolia L.*

Morinda coreia Buch.-Ham.

Moringa oleifera Lam.*

Mucuna pruriens var. utilis (Wall. ex Wight) L.H.Bailey -

Murraya koenigii (L.) Spreng.*

Myristica fragrans Houtt.*

Nardostachys jatamansi (D.Don) DC.

Nelumbo nucifera Gaertn.*

Neopicrorhiza scrophulariiflora (Pennell)

D. Y. Hong

Ocimum americanum $\mathrm{L}$.

Ocimum basilicum L.*

Ocimum gratissimum L.*

Ocimum tenuiflorum L.*

Onosma bracteata Wall.

Onosma hispida Wall. ex G.Don

Operculina turpethum (L.) Silva Manso

Oroxylum indicum (L.) Kurz

Paederia foetida $\mathrm{L}$.

Parmelia perlata (Huds.) Ach

Pedalium murex L.

Peganum harmala L.

Phyllanthus amarus Schum. \& Thonn.

Phyllanthus emblica L.

Phyllanthus maderaspatensis L.

Picrorhiza kurroa Royle ex Benth.
Karnataka (VU 1999)

$-$

$-$

$-$

$-$

Orissa (EN 2007), Tripura (NT 2016), West Bengal (EN 2007)

LC

$-$

West Bengal (VU 2007)

$-$

$-$

Chhattisgarh (NT 2003), Madhya Pradesh (NT 2003), West Bengal (EN 2007)

$-$

$-$

CR Sikkim (VU 2014)

-

- $\quad$ Sikkim (VU 2014)

$-$

$-\quad-$

- $\quad$ Rajasthan (VU 2007)

- $\quad-$

$-$

$-$

- $\quad$ Chhattisgarh (NT 2003), Karnataka (VU 1999), Kerala (EN 1999), Madhya Pradesh (NT 2003), Maharashtra (EN 2001), Orissa (VU 2007), Tamil Nadu (NT 1998)

Andhra Pradesh (VU 2001), Arunachal Pradesh (VU 2003), Assam (VU 2003), Chhatisgarh (VU 2003), Himachal Pradesh (NE 2010), Karnataka (VU 1996), Kerala (EN 1996), Madhya Pradesh (VU 2003), Maharashtra (EN 2001), Meghalaya (VU 2003), Nagaland (VU 2015), Orissa (EN 2007), Rajasthan (EN 2007), Sikkim (VU 2003, NT 2014), Tripura (NT 2016)

$-$

Andhra Pradesh (NT 2001), Orissa (VU 2007)

$-$

Rajasthan (VU 2007)

$-$

- $\quad$ Chhatisgarh (VU 2003), Madhya Pradesh (VU 2003)

$-$

- $\quad$ Arunachal Pradesh (EN 2003), Himachal Pradesh (EN 2003, EN2010), Jammu and Kashmir (EN 2003), Sikkim (VU 2003), Uttarakhand (VU 2003), West Bengal (CR 2007) 
Table 3 continued

\begin{tabular}{|c|c|c|}
\hline \multirow[t]{2}{*}{ Species } & \multicolumn{2}{|c|}{ Threat status } \\
\hline & $\begin{array}{l}\text { IUCN } \\
\text { threat } \\
\text { category }\end{array}$ & $\begin{array}{l}\text { CAMP exercise threat category } \\
\text { [State (threat category and year of CAMP exercise)] }\end{array}$ \\
\hline Pinus roxburghii Sarg. & - & - \\
\hline Piper chaba Hunter $=$ Piper retrofractum Vahl & - & - \\
\hline Piper longum L.* & - & $\begin{array}{l}\text { Chhatisgarh (VU 2003), Kerala (NT1995), Orissa (EN 2007), } \\
\text { Tamil Nadu (EN 1995) }\end{array}$ \\
\hline $\begin{array}{l}\text { Pistacia integerrima J.L. Stewart ex Brandis = Pistacia } \\
\text { chinensis subsp. integerrima (J. L. Stewart ex Brandis) } \\
\text { Rech. f. }\end{array}$ & - & - \\
\hline Plantago ovata Forssk.* & - & - \\
\hline Pluchea lanceolata (DC.) C.B.Clarke & - & Madhya Pradesh (NT 2003) \\
\hline Plumbago indica L.* & - & Andhra Pradesh (EN 2001), \\
\hline Plumbago zeylanica L. & - & $\begin{array}{l}\text { Chhatisgarh (VU 2003), Madhya Pradesh (VU 2003), } \\
\text { Rajasthan (VU 2007) }\end{array}$ \\
\hline Polygonatum cirrhifolium (Wall.) Royle & - & $\begin{array}{l}\text { Himachal Pradesh (EN 2003, EN 2010), Sikkim (VU 2014), } \\
\text { Uttarakhand (VU 2003) }\end{array}$ \\
\hline Pongamia pinnata (L.) Pierre* & $\mathrm{LC}$ & - \\
\hline Premna corymbosa Rottler \& Willd. & - & - \\
\hline Premna serratifolia $\mathrm{L}$. & - & - \\
\hline Prunus armeniaca L.* & - & - \\
\hline Prunus cerasoides Buch.-Ham. ex D.Don & - & - \\
\hline Pseudarthria viscida (L.) Wight \& Arn. & & $\begin{array}{l}\text { Karnataka (VU 1999), Kerala (VU 1999), Tamil Nadu (NT } \\
\text { 1998) }\end{array}$ \\
\hline Pterocarpus marsupium Roxb. & NT & $\begin{array}{l}\text { Chhattisgarh (VU 2003), Madhya Pradesh (VU 2003), } \\
\text { Maharashtra (VU 2001), Orissa (EN 2007), Rajasthan (CR } \\
\text { 2007), West Bengal (EN 2007) }\end{array}$ \\
\hline Pterocarpus santalinus L.f & NT & Andhra Pradesh (CR 2001), Tamil Nadu (CR 1998) \\
\hline Pueraria tuberosa (Roxb. ex Willd.) DC. & - & $\begin{array}{l}\text { Andhra Pradesh (NT 2001), Karnataka (CR 1999), Kerala } \\
\text { (VU 1999), Madhya Pradesh (EN 2006), Maharashtra (VU } \\
\text { 2001), Orissa (VU 2007), Rajasthan (VU 2007), Tamil } \\
\text { Nadu (VU 1998) }\end{array}$ \\
\hline Punica granatum L. & - & - \\
\hline Quercus infectoria G. Olivier & - & - \\
\hline Rauvolfia serpentina (L.) Benth. ex Kurz & - & $\begin{array}{l}\text { Andhra Pradesh (CR 2001), Arunachal Pradesh (CR 2003), } \\
\text { Assam (VU 2003), Chhatisgarh (CR 2003), Himachal } \\
\text { Pradesh (CR 2003), Jammu and Kashmir (VU 2003), } \\
\text { Karnataka (EN 1995), Kerala (EN 1995), Madhya Pradesh } \\
\text { (VU 2003), Maharashtra (CR 2001), Meghalaya (VU } \\
\text { 2003), Nagaland (DD 2015), Orissa (EN 2007), Tamil } \\
\text { Nadu (VU 1995), Tripura (VU 2016), Uttarakhand (VU } \\
\text { 2003), West Bengal (EN 2007) }\end{array}$ \\
\hline Rheum australe D.Don & - & - \\
\hline Rhododendron arboretum $\mathrm{Sm}$. & - & - \\
\hline Rubia cordifolia $\mathrm{L}$. & - & $\begin{array}{l}\text { Andhra Pradesh (VU 2001), Chhatisgarh (VU 2003), Madhya } \\
\text { Pradesh (VU 2003), Maharashtra (VU 2001) }\end{array}$ \\
\hline Salacia reticulata Wight & - & Karnataka (EN 1999) \\
\hline Salix caprea $\mathrm{L}$. & - & - \\
\hline
\end{tabular}


Table 3 continued

Species

Sapindus mukorossi Gaertn.*

Saraca asoca (Roxb.) Willd.

Saussurea costus (Falc.) Lipsch.*

Scindapsus officinalis (Roxb.) Schott

Semecarpus anacardium L.f.

Senna alexandrina Mill.*

Senna auriculata (L.) Roxb.

Senna occidentalis (L.) Link

Senna tora (L.) Roxb.

Shorea robusta Gaertn.

Sida acuta Burm.f.

Sida cordifolia L.

Sida rhombifolia $\mathrm{L}$.

Smilax china L.

Solanum anguivi Lam.

Solanum nigrum L. = Solanum americanum Mill.

Solanum virginianum $\mathrm{L}$.

Spermacoce hispida $\mathrm{L}$.

Sphaeranthus indicus L.

Stereospermum chelonoides (L.f.) DC.

Stereospermum tetargonum DC.

Strobilanthes ciliata Nees

Strychnos nux-vomica A.W.Hill

Strychnos potatorum L.f.

Swertia chirayita (Roxb.) Buch.-Ham. ex C.B.Clarke

Symplocos cochinchinensis (Lour.) S. Moore

Symplocos racemosa Roxb.

Syzygium cumini (L.) Skeels*

Tamarix gallica $\mathrm{L}$.

Tamarix indica Willd

Tanacetum cinerariifolium (Trevir.) Sch. Bip.*

Taxus wallichiana Zucc.

Threat status

IUCN CAMP exercise threat category

threat [State (threat category and year of CAMP exercise)]

category

VU

$-$

- $\quad$ Andhra Pradesh (EN 2001), Karnataka (EN 1995), Maharashtra (EN 2001), Orissa (CR 2007), Tripura (CR 2016)

CR Jammu and Kashmir (CR 2003)

- $\quad$ Orissa (VU 2007)

- $\quad-$

- $\quad-$

$-$

-

- $\quad-$

LR/lc Andhra Pradesh (NT 2001)

- $\quad$ -

$-$

$-$

$-$

$-$

$-\quad-$

$-$

$-$

LC -

- $\quad$ Chhatisgarh (NT 2003), Madhya Pradesh (NT 2003)

$-\quad-$

$-\quad-$

- $\quad$ Chhatisgarh (VU 2003)

- $\quad$ Orissa (VU 2007)

- $\quad$ Arunachal Pradesh (VU 2003), Himachal Pradesh (CR 2003, CR 2010), Jammu and Kashmir (CR 2003), Meghalaya (VU 2003), Sikkim (VU 2003, EN 2014), Uttarakhand (EN 2003), West Bengal (CR 2007)

-

- $\quad$ Karnataka (VU 1996), Madhya Pradesh (VU 2006), Maharashtra (VU 2001), Nagaland (NT 2015), Orissa (CR 2007), Tamil Nadu (NT 1996)

$-$

- $\quad-$

$-$

- $\quad-$

EN Arunachal Pradesh (EN 2003), Himachal Pradesh (EN 2003, EN 2010), Jammu and Kashmir (EN 2003), Meghalaya (CR 2003), Sikkim (EN 2003), Uttarakhand (EN 2003), West Bengal (CR 2007) 
Table 3 continued

Species Threat status

IUCN CAMP exercise threat category

threat [State (threat category and year of CAMP exercise)] category

Tecomella undulata (Sm.) Seem.

\begin{tabular}{|c|c|}
\hline- & Rajasthan (EN 2007) \\
\hline- & - \\
\hline- & - \\
\hline- & $\begin{array}{l}\text { Chhatisgarh (NT 2003), Karnataka (NT 1997), Kerala (NT } \\
\text { 1997), Madhya Pradesh (NT 2003), Maharashtra (NT } \\
\text { 2001), Rajasthan (VU 2007) }\end{array}$ \\
\hline- & - \\
\hline- & Chhatisgarh (VU 2003), Madhya Pradesh (VU 2003) \\
\hline- & - \\
\hline- & $\begin{array}{l}\text { Karnataka (VU 1999), Kerala (NT 1999), Maharashtra (NT } \\
\text { 2001) }\end{array}$ \\
\hline- & - \\
\hline- & - \\
\hline- & - \\
\hline- & - \\
\hline- & Andhra Pradesh (NT 2001), Madhya Pradesh (NT 2006) \\
\hline- & Sikkim (DD 2015) \\
\hline $\mathrm{LC}$ & $\begin{array}{l}\text { Chhattisgarh (VU 2003), Himachal Pradesh (NE 2010), } \\
\text { Madhya Pradesh (VU 2003), Orissa (EN 2007) }\end{array}$ \\
\hline- & $\begin{array}{l}\text { Arunachal Pradesh (VU 2003), Himachal Pradesh (VU } \\
\text { 2003), Jammu and Kashmir (VU 2003), Meghalaya (VU } \\
\text { 2003), Nagaland (VU 2015), Sikkim (VU 2003, VU 2014), } \\
\text { Uttarakhand (VU 2003) }\end{array}$ \\
\hline- & - \\
\hline- & - \\
\hline- & - \\
\hline- & - \\
\hline- & - \\
\hline LC & $\begin{array}{l}\text { Himachal Pradesh (EN 2003, EN 2010), Jammu and Kashmir } \\
\text { (VU 2003), Nagaland (VU 2015), Orissa (VU 2007), } \\
\text { Uttarakhand (VU 2003) }\end{array}$ \\
\hline $\mathrm{DD}$ & - \\
\hline- & - \\
\hline
\end{tabular}

Terminalia bellirica (Gaertn.) Roxb.

Terminalia chebula Retz.

Tinospora cordifolia (Willd.) Miers

Tinospora sinensis (Lour.) Merr.

Tragia involucrata $\mathrm{L}$.

Trianthema decandra $\mathrm{L}$.

Tribulus lanuginosus L.

Tribulus terrestris $\mathrm{L}$.

Trichosanthes cucumerina L.

Trillidium govanianum Wall. ex D.Don

Uraria picta (Jacq.) DC.

Valeriana jatamansi Jones

Viola pilosa Blume

Vitex negundo L.*

Withania somnifera Dunal *

Woodfordia fruticosa (L.) Kurz

Wrightia tinctoria R.Br.

Zanthoxylum armatum DC.

Zingiber zerumbet (L.) Roscoe ex Sm.

Ziziphus mauritiana Lam.

Rajasthan (EN 2007)

Chhatisgarh (NT 2003), Karnataka (NT 1997), Kerala (NT 1997), Madhya Pradesh (NT 2003), Maharashtra (NT 2001), Rajasthan (VU 2007)

Karnataka (VU 1999), Kerala (NT 1999), Maharashtra (NT 2001)

EX: Extinct; CR: Critically Endangered; EN: Endangered; VU: Vulnerable; LR: Lower Risk; LR: Lower Risk; NT: Near threatened; LC: Least concern; DD: Data deficient

IUCN Red list under medicine for human and veterinary group is used

(Source: Goraya and Ved 2017)

*species which are in cultivation

pertaining to the medicinal plants (NMPB 2020a). Thereafter in 2006-07 and 2015, NMPB commissioned the nation-wide study through the ICFRE in strategic partnership with FRLHT with the major objective to assess the status of demand and supply of medicinal plants in India for the said period (Goraya and Ved 2017). 
In a span of ten years (2005-14) tremendous increase in demand and supply of the medicinal plants is recorded (Goraya and Ved 2017). A 62\% increase in annual demand of herbal raw drugs was observed for the year 2014-15 over the demand estimated for the year 2005-06 (Ved and Goraya 2008). The volume of botanical drugs exported increased from 56,500 Metric Tonne (MT) in 2005-06 to $1,34,500$ MT in 2014-15. About seven times higher (10,680 million in 2005-06 to 70,000 million in 2014-15) estimated trade value of herbal drugs was registered in 10 years. The trade value of herbal raw drugs consumed by the domestic herbal industry has also registered more than two fold increase as per the latest estimates during 2014-15. A total of 242 species are enlisted as high commercial demand (>100 MT/year) during 2014-15 (Table 3) in contrast to 178 species in 2005-06 (Ved and Goraya 2008). Out of 242 species $114(47 \%)$ are collected from forests, 59 species (25\%) are wild and collected from landscapes outside forests, 54 species $(22 \%)$ are obtained from cultivation and 15 species $(6 \%)$ are imported. A total of 1,389 botanical entities corresponding to 960 plant species were enlisted under trade in the study conducted by NMPB (Goraya and Ved 2017). India's domestic herbal industry is represented by 8,610 licensed herbal units (Ayurveda-7,494, Unani-421, Siddha-328 and Homeopathy-367) spread across different states in the country. Uttar Pradesh has the highest units of 2,247 followed by Kerala (905), Maharashtra (705), Tamil Nadu (662), Madhya Pradesh (633), Andhra Pradesh (610), Gujarat (495), Haryana (300), Punjab (284), Bihar (281), West Bengal (277), Rajasthan (268), Uttarakhand (240), Karnataka (177), Orissa (160), Himachal Pradesh (138), Delhi (63), Assam (53), Pondicherry (41), Nagaland (34), Chhattisgarh (31), Jammu and Kashmir (15), Daman and Diu (10), Goa (7), Dadra and Nagar Haveli (5), Chandigarh (1), Meghalaya (1) and Sikkim (1) (Goraya and Ved 2017).

\section{National efforts towards conservation of medicinal plants}

The Government of India (GoI) took due cognizance for medicinal plants since early 1990s and several activities have been undertaken to protect and conserve these species both in situ (biosphere reserves, national parks, wild life sanctuaries, sacred groves etc.) and ex situ (botanical gardens, field gene banks, seed gene banks, in vitro gene banks and cryogenebanks). For in situ conservation, of the total geographical area of India about 16.5 million ha $(5.02 \%)$ is under protected areas and 70.8 million ha $(21.54 \%)$ is under forests. In India, a total of 870 protected areas are earmarked which include 104 national parks, 551 wildlife sanctuaries, 127 community reserves, 88 conservation reserves (WIIENVIS 2019). Further, an estimated 100,000 - 150,000 sacred groves are present in India (Kandari et al. 2014). For ex situ conservation several institutes/organizations have been established by GoI, especially to undertake research on medicinal plants viz., Central Council for Research in Ayurvedic Sciences (CCRAS), Central Council for Research in Homoeopathy (CCRH), Central Council for Research in Siddha (CCRS) and Central Council for Research in Unani Medicine (CCRUM) under Ministry of Ayurveda, Yoga and Naturopathy, Unani, Siddha and Homoeopathy (AYUSH), Botanical Survey of India (BSI) and its regional circles and experimental gardens at different geographic regions of India, Kerala State Council for Science, Technology and Environment (KSCSTE)Jawaharlal Nehru Tropical Botanic Garden and Research Institute (KSCSTE - JNTBGRI, formerly TBGRI), Indian Council of Forestry Research and Education (ICFRE), Council of Scientific and Industrial Research (CSIR) institutes i.e., Central Drug Research Institute (CDRI), Central Institute of Medicinal and Aromatic Plants (CIMAP), National Botanical Research Institute (NBRI) and the Regional Research Laboratories (RRLs), Institute of Himalayan Bioresource Technology; Institutes under the Indian Council of Agricultural Research (ICAR) namely ICAR-National Bureau of Plant Genetic Research (ICAR-NBPGR), ICAR-Directorate of Medicinal and Aromatic Plants (ICAR-DMAPR) and ICAR-Indian Institute of Horticultural Research (ICAR-IIHR), ICAR-All India Network Research Project on Medicinal and Aromatic Plants (AINRP MAPs). In addition, several other government and non-government organizations, industries, ayurvedic practitioners are also involved in conservation and cultivation of these medicinal plants (Bhattacharyya et al. 2006).

A network of four National Gene Banks for Medicinal and Aromatic Plants (GEBMAP) were set up in 1993 at (1) ICAR-NBPGR (2) KSCSTE JNTBGRI, (3) CSIR-CIMAP, and (4) Regional 
Research Laboratory (RRL) Jammu (added later) under the G-15 GEBMAP program with Department of Biotechnology (DBT), Ministry of Science and Technology, GoI, as nodal agency. This has not only given better focus and thrust especially on collection and conservation of medicinally important threatened species but also helped in consolidating the ongoing efforts in the country (Sharma and Pandey 2013; Sharma et al. 2019, 2020). JNTBGRI herbal garden has a collection of 1,200 taxa herbals in an area of 10 acres (KSCSTE-JNTBGRI 2019). At CSIR-CIMAP, a total of 3,334 accessions in seed bank (2,476 accessions) and field genebank (868 accessions) are being maintained (Rajpurohit and Jhang 2015).

ICAR-NBPGR is the nodal agency for all the activities introduction, collection, conservation, documentation, evaluation and distribution of plant genetic resources (PGR) in the country. It has 10 regional stations located in different agro-climatic zones of the country and the 59 National Active Germplasm Sites (NAGS) which are based at ICAR institutes (specific crops groups) and are assigned with multiplication, evaluation, conservation of active collections of germplasm and their distribution to users both at the national and international levels. In addition, several ICAR institutes, State Agricultural Universities and other stakeholders are also linked to the network (Singh et al. 2016). The National Gene Bank of ICAR-NBPGR has four types of conservation facilities i.e., seed gene bank, cryogenebank, in vitro gene bank and field genebank. At ICAR-NBPGR, 8,071 accessions of MAPs in seed genebank, 178 accessions in in vitro genebank and 1,041 accessions in cryogenebank are being conserved (Singh and Pandey 2019; Sharma et al. 2020).

The GoI has also established NMPB in 2000 under Ministry of AYUSH, and provided funds for research on medicinal plants. In addition to NMPB, DBT and Department of Science and Technology (DST) have also supported researchers to undertake research on medicinal plants. Recently (2019) NMPB prioritized 32 medicinal plants for conservation (Aconitum ferox Wall ex Ser., Aconitum heterophyllum Wall., Aegle marmelos (L.) Corr., Andrographis paniculata, Asparagus racemosus (Burm.) Nees, Bacopa monnieri (L.) Wettst., Berberis aristata DC., Cassia angustifolia M Vahl. = Senna alexandrina Mill., Chlorophytum borivillianum Sant., Coleus barbatus Benth., Commiphora wightii (Arn.) Bhandari, Crocus sativus L.,
Embelia ribes Burm.f., Emblica officinalis Gaertn. = Phyllanthus emblica L, Garcinia indica Choisy, Gloriosa superb L., Glycyrrhiza glabra L., Gymnema sylvestre (Retz.) R. Br., Nardostachys jatamansi (Retz.) R. Br., Ocimum sanctum L., Phyllanthus amarus Schum. \& Thonn., Picrorhiza kurroa Royle ex Benth., Piper longum L, Plantago ovate Forsk., Rauvolfia serpentina Benth. ex Kurz., Santalum album L., Saraca asoca (Roxb.) De Wilde, Saussurea costus (Falc.) Lipsch, Solanum nigrum L., Swertia chirata Roxb. ex Flem., Tinospora cordifolia Miers., Withania somnifera Dunal (NMPB 2020b). NMPB has also taken initiatives for in situ conservation of medicinal plants through establishment of 72 Medicinal Plants Conservation and Development Areas (MPCDAs) across 13 states of the country, covering an area of 10,935 ha in forest areas (Biswas et al. 2017) and 36 State Medicinal Plant Boards in State/UT. FRLHT in collaboration with the State Forest Departments and with financial support of Danish International Development Aid (DANIDA), Netherlands, and United Nations Development Programme (UNDP) has established 108 Medicinal Plant Conservation Areas (MPCAs) across 13 states and 18 Medicinal Plants Conservation Parks (MPCPs) in southern India for focusing on conservation of prioritized wild medicinal plants occurring in different regions of the country (FRLHTENVIS 2016b). In 2012, DBT initiated a mega-network programme on 'Preventing extinction and improving the conservation status of threatened plants through application of biotechnological tools and conserved 100 threatened species of India (Barik et al. 2018). Under this project 115 species were reintroduced, macropropagation protocols were standardized for 106 species and micropropagation protocols were standardized for 76 species (Barik et al. 2018).

\section{Regulatory and policy framework in India}

The medicinal plant species in trade (both domestic and international) are sourced from many different agro-climatic zones in the country from the wild, and only a very small number of species are cultivated (Pareek et al. 2005; Sharma and Pandey 2013). Besides these, there is no data documented regarding the consumption levels of botanicals by the noncodified and non-commercial folk healthcare 
Table 4 List of plants notified by the Central Government, in consultation with the State Government under Sect. 38 of India's Biological Diversity Act, 2002 ( Source: The Gazette of India: Extraordinary, Ministry of Environment and Forests of each state)

\begin{tabular}{lll}
\hline State/Union & Listed species & Notification date in \\
Territory & & Gazette of India \\
\hline
\end{tabular}

Assam

Andaman and Nicobar Islands

Bihar

Goa

Himachal Pradesh

Jammu \& Kashmir

Kerala

Karnataka

Manipur

Madhya Pradesh

Meghalaya
Cycas pectinata Buch.-Ham., Magnolia cathcartii (Hook.f. \& Thomson) Noot., Magnolia manni (King) Figlar, Mesua assamica (King \& Prain) Kosterm., Paphiopedilium spicerianum (Rchb.f.) Pfitzer, Vatica lanceaefolia (Roxburgh) Blume

Artabotrys nicobarianus D.Das, Calamus nicobaricus Becc., Korthalsia rogersii Becc., Mangifera andamanica King, Ophioglossum pendulum L.

Chrysopogon hamiltonii (Hook.f.) Haines

Ceropegia fantastica Sedgw., Phyllanthus talbotii Sedgw.

Aconitum deinorrhizum Stapf., Aconitum heterophyllum Wall. ex Royle., Aconitum violaceum Jacquem. ex Stapf, Dactylorhiza hatagirea (D.Don) Soó, Eremostachys superba Royle ex Benth., Jasminum parkeri Dunn., Nardostachys grandiflora DC, Taxus wallichiana Zucc.

Aconitum chasmanthum Stapf. ex Holmes, Aconitum deinorrhizum Stapf., Aconitum heterophyllum Wall. ex Royle., Aconitum kasmiricum Stapf Ex. Coventry., Aconitum violaceum Jacquem. ex Stapf,, Betula utilis D. Don, Dactylorhiza hatagirea (D.Don) Soó., Eremostachys superba Royle ex Benth., Gentiana ornata Wall. Ex. Griseb., Gentiana kurroo Royle, Lagotis cashmeriana (Royle) Rupr., Meconopsis latifolia (Prain) Prain., Meconopsis aculeata Royle, Podophyllum hexandrum Royle, Picrorrhiza kurroa Royle Ex. Benth., Taxus wallichiana Zucc., Saussurea costus (Falc.) Lipsch., Saussurea medusa Maxim., Saussurea simpsoniana (Fielding \& Gardner) Lipsch., Sophora moorcroftiana Benth. Ex. Baker

Adenosma malabaricum Hook.f., Agasthiyamalaia pauciflora (Bedd.) S.Rajkumar \& Janarth. (= Poeciloneuron pauciflorum Bedd.), Aglaia malabarica Sasidh. Anacolosa densiflora Bedd., Atuna indica (Bedd.) Kosterm., Calliandra cynometroides Bedd., Cinnamomum travancoricum Gamble, Dialium travancoricum Bourd., Dimorphocalyx beddomei (Benth.) Airy Shaw, Dipterocarpus bourdillonii Brandis, Elaeocarpus venustus Bedd., Garcinia imbertii Bourd., Haplothismia exannulata Airy Shaw, Humboldtia bourdillonii Prain, Humboldtia trijuga (Bedd.) M. Mohanan, Janakia arayalpathra J. Joseph \& Chandras., Madhuca bourdillonii (Gamble) H.J.Lam, Memecylon sisparense Gamble, Paphiopedilum druryi (Bedd.) Stein, Salacia malabarica Gamble, Syzygium palghatense Gamble, Syzygium periyarensis Augustine \& Sasidh., Toxocarpus beddomei Gamble, Toxocarpus palghatensis Gamble, Vanda thwaitesii Hook.f., Vanda wightii Rchb.f

Barleria grandiflora Dalzell., Beaumontia jerdoniana Wight, Ceropegia decaisneana Wight, Cinnamomum goaense Kosterm., Holigarna beddomei Hook.f., Hopea canarensis Hole, Impatiens mysorensis Roth, Impatiens raziana Bhaskar \& Razi, Justicia nilgherrensis (Nees) Wall. ex C.B.Clarke, Madhuca insignis (Radlk.) H.J.Lam, Phyllanthus talbotii Sedgw., Salacia malabarica Gamble, Toxocarpus concanensis Hook.f., Toxocarpus palghatensis Gamble, Vanda thwaitesii Hook.f., Vanda wightii Rchb.f

Kalanchoe rosea Clarke, Lilium davidii Duch. ex Elwes, Malus baccata (L.) Borkh., Rhododendron macabeanum Watt ex Balf.f., Rhododendron wattii Cowan

Alsophila balakrishnanii (Dixit \& Tripathi) Dixit = Cyathea gigantea (Wall.ex Hook.) Holtt., Ficus cupulata Haines, Jasminum brevipetiolatum Duthie

Aquilaria khasiana Hallier f., Gymnocladus assamicus P.C.Kanjilal, Ilex khasiana Purkay., Jasminum adenophyllum Wall. ex C.B.Clarke, Nepenthes khasiana Hook.f., Nymphaea tetragona Georgi
August 3, 2018

October 26, 2012

February 8, 2011

March 31, 2010

March 19, 2009

August 3, 2018

April 23, 2009

November 4, 2020

October 29, 2012

June 7, 2010

September 30, 2009 
Table 4 continued

\begin{tabular}{|c|c|c|}
\hline $\begin{array}{l}\text { State/Union } \\
\text { Territory }\end{array}$ & Listed species & $\begin{array}{l}\text { Notification date in } \\
\text { Gazette of India }\end{array}$ \\
\hline
\end{tabular}

\begin{tabular}{|c|c|}
\hline Mizoram & $\begin{array}{l}\text { Aquilaria malaccensis Lam.,Cinnamomum aromaticum Nees }=\text { C. cassia (L.) } \\
\text { J.Presl., Dalbergia pinnata var. acaciifolia (Dalzell) Thoth., Hydnocarpus kurzii } \\
\text { (King) Warb., Jasminum wengeri C.E.C.Fisch., Mantisia wengeri C.E.C.Fisch., } \\
\text { Paphiopedilum spicerianum (Rchb.f.) Pfitzer, Paphiopedilum villosum (Lindl.) } \\
\text { Stein }\end{array}$ \\
\hline Orissa & Lasiococca comberi Haines \\
\hline Punjab & $\begin{array}{l}\text { Alysicarpus bupleurifolius var. hybridus DC., Ceropegia bulbosa var.lushii } \\
\text { (Graham) Hook.f. = Ceropegia lushii Graham), Hibiscus hoshiarpurensis T.K.Paul } \\
\text { \& M.P.Nayar, Ophioglossum gramineum Willd., Ophioglossum polyphyllum A. } \\
\text { Braun ex Schub., Tecomella undulata (Sm.) Seem., Withania coagulans (Stocks) } \\
\text { Dunal, Anogeissus sericea var. nummularia King ex Duthie }\end{array}$ \\
\hline Tamil Nadu & $\begin{array}{l}\text { Belosynapsis kewensis Hassk, Byrosphyllum tetrandrum (Bedd.) J.Hk. ex Bedd., } \\
\text { Cinnamomum walaiwarense Kosterm., Didymocarpus missionis Wall. ex R.Br., } \\
\text { Dimorphocalyx beddomei (Benth.) Airy Shaw, Diospyros barberi Ramaswami, } \\
\text { Diospyros humilis (R.Br.) F.Muell., Drypetes porteri (Gamble) Pax \& K.Hoffm., } \\
\text { Elaeocarpus blascoi Weibel, Elaeocarpus venustus Bedd., Hoya kanyakumariana } \\
\text { A.N.Henry \& Swamin., Impatiens auriculata Wight, Janakia arayalpathra J. } \\
\text { Joseph \& Chandras., Memecylon sisparense Gamble, Paphiopedilum druryi } \\
\text { (Bedd.) Stein, Phyllanthus anamalayanus (Gamble) G.L.Webster } \\
\text { (= Pseudoglochidion anamalayapum Gamble), Popowia beddomeana Hook.f. \& } \\
\text { Thomson, Sonerila pulneyensis Gamble, Symplocos oligandra Bedd., Syzygium } \\
\text { beddomei (Duthie) Chithra, Syzygium courtallense (Gamble) Alston, Syzygium } \\
\text { gambleanum Rathakr. \& V. Chithra, Toxocarpus beddomei Gamble }\end{array}$ \\
\hline
\end{tabular}

Tripura Stichoneuron membranaceum Hook.f

Uttarakhand Aconitum balfourii Stapf, Aconitum heterophyllum Wall. ex Royle, Aconitum violaceum Jacquem. ex Stapf, Butea pellita Prain, Cyathea spinulosa Wall. ex Hook., Diplomeris hirsuta (Lindl.) Lindl., Eremostachys superba Royle ex Benth., Gentiana kurroo Royle, Indopiptadenia oudhensis (Brandis) Brenan, Nardostachys grandiflora DC., Pinguicula alpina L., Schrebera swietenioides Roxb., Trachycarpus takil Becc., Turpinia nepalensis Wall

West Bengal Hibiscus fragrans Roxburgh, Streptocaulon sylvestre Wight

September 30, 2009

February 4, 2014

March 3, 2011

February 3, 2011

April 15,, 2019

April 7, 2010

Authority and appropriate synonyms as per The Plant List (www.theplantlist.org), International Plant Names Index (IPNI; www.ipni. org) and India Biodiversity Portal (indiabiodiversity.org)

traditions, based primarily on ecosystem and regionspecific plant material practiced by around one million folk practitioners and $\sim 140$ millions of rural households in the country. It is important to note that of 242 species in high commercial demand ( $>100$ MT per year), $72 \%$ of these species is sourced largely from wild plants as roots, bark, wood stem or the whole plant. This poses a major concern as it affects the survival of these species. Unsustainable collecting activities coupled with limited or no efforts of replenishment/cultivation, long duration life cycle of plants, climate change and developmental activities have led to rapid decline in the availability of many medicinal plant species in the country during the last three decades. This has culminated into a large number of species falling in to threatened group at country as well as global level. In order to minimize this, the GoI has developed few regulatory frameworks and policies viz.,

- Indian Forest Act, 1927;

- Panchayat Raj Act, 1933;

- The West Bengal Forest Produce Transit Rules, 1955;

- Andaman and Nicobar Island Forest Produce Transit Rules, 1966;

- Wildlife (Protection) Act, 1972 (Amended 2003) Also 2006;

- The Convention on International Trade in Endangered Species of Wild Fauna and Flora, 1975;

- Kerala Forest Produce Transit Rules, 1975; 
- The Forest (Conservation) Act (Amended), 1980;

- Environment Protection Act, 1986;

- National Forest Policy, 1988;

- Negative list of Plants for Exports and Imports (Revised), 1994;

- Panchayat (Extension to Scheduled Areas)(PESA) Act, 1996;

- National Biodiversity Strategy and Action Plan, 2002;

- The Biological Diversity Act, 2002;

- Biological Diversity Rules, 2004;

- Negative list of MAP Collection from Wild, 2004;

- Scheduled Tribes and Other Traditional Forest Dwellers Act (FRA), 2006;

- Maharashtra Forests Rules, 2014, The HP Forest Produce Transit (Land Route) Rules, 2013;

- Export Import Policy, 2015-20;

- National Wildlife Action Plan 2017-31;

As per Sect. 38 of the Biological Diversity Act, 2002, the Central Government, in consultation with the Government of states, notifies the plants which are on the verge of extinction (Table 4). The NMPB has developed several policies, strategies and programs for conservation, proper harvesting, cost-effective cultivation, research and development, processing, marketing of raw material to promote and develop medicinal plants sector. Recently a Memorandum of Understanding (MoU) has been signed between ICAR-NBPGR and NMPB on July 6, 2020 for safe conservation of Medicinal and Aromatic Plants Genetic Resources for long-term storage at the National Gene Bank of ICAR-NBPGR (ICARNBPGR 2020).

In addition to national policy framework, India has also signed few international agreements:

(i) Convention on International Trade in Endangered Species of Wild Fauna and Flora (CITES), an international agreement between governments which aims to ensure that international trade of wild animals and plants does not lead to any threat on their survival. There are about 5,000 species of animals and 28,000 species of plants that are protected by CITES against over-exploitation through international trade. The Ministry of Environment, Forest and Climate Change, GoI, has notified a list of 113 species (CITES 2017), the export of which requires 'Certificate of Cultivation' or 'Legal Procurement Certificate' from the designated authorities of the Forest Departments. At present twelve of the Indian medicinal plants are included in the appendices of CITES viz., Appendix I (Saussurea costus), Appendix II (Aquilaria malaccensis Lam. = A. agalocha Roxb.ex DC., Cycas bedomii Dyer., Dioscorea deltoidea Wall. Ex Kunth, Rauvolfia serpentina, Cibotium barometz Link., Podophyllum hexandrum Royle, Pterocarpus santalinus L.F., Nardostachys grandiflora DC., Nepenthes khasiana Hook. f., Picrorhiza kurrooa, Taxus wallichiana Zucc.).

(ii) The EXIM Policy-Plants Prohibited for Exports. Under this export of 29 plants (Aconitum species, Aquilaria malaccensis, Coscinium fenestratum (Gaertn.) Coleb., Coptis teeta Wall., Cyatheaceae species, Cycas beddomei, Cycadaceae species, Dioscorea deltoidea, Dactylorhiza hatagirea (DON.) SOO, Euphorbia species, Frerea indica Dalz, Gentiana kurroo Royle., Gnetum species, Podophyllum hexandrum, Kaempferia galanga L., Nardostachys grandiflora, Nepenthes khasiana, Orchidaceae species, Panax pseudoginseng Wall., Paphiopedilium species, Picrorhiza kurrooa, Pterocarpus santalinus, Rauvolfia serpentina, Renanthera imschootiana Rolfe., Saussurea costus, Swertia chirata, Taxus wallichiana, Vanda coerulea Griff. Ex Lindl.), plant portions and their derivatives and extracts as such obtained from the wild except the formulations made there from, is prohibited.

(iii) Foreign Trade under ITC (HS) Codes and its Limitations.

\section{Reintroduction of threatened plants in natural habitat}

Reintroduction of threatened plants is the approach of re-establishment into an area suitable for its growth or from where it has become threatened. Through microor macropropagation, such plants have to be produced in large numbers to reintroduce in nature especially. The basic idea of this approach is to establish a selfsustaining population for conservation purposes. A species is said to be 'recovered', once the factors that initially led to its listing in threatened species are remedied and protection is no longer required. This approach has been successfully applied in India as a part of conservation efforts of threatened plants i.e., Vanda coerulea Griff ex. Lindl. (Seeni and Latha 2000), Syzygium travancorium Gamble (Anand 2003), 
Decalpis arayalpathra J. Joseph \& V. Chandrasekaran, Mahonia leschenaultia (Wallich ex Wight $\&$ Arnott) Takeda = Berberis napaulensis, Heracleum candolleanum (WT. \& ARN.) GAMBLE, Calophyllum apetalum Willd. and Blepharistemma serratum (Densst.) Suresh = Blepharistemma membranifolia (Krishnan et al. 2011), Ceropegia fantastica Sedgtvick (Ravikanth et al. 2018). In recent years, the DBT has initiated a several species-specific recovery programmes targeting 156 highly threatened species of the country during the past three decades. These species belong to 101 genera and 64 families, and comprise herbs (50), trees (42), orchids (24), shrubs (14), climbers (14), bamboos (3), palms (3), rattans (3), cycads (2), and tree fern (1) (DBT 2019).

\section{Way Forward}

- Every plant on this planet is known to possess one or the medicinal properties, but all these plants can't be classified as medicinal plants. For example, Abelmoschus esculentus (L.) Moench., Allium cepa L., A. sativum L., Amaranthus spinosus L., A. tricolor L., Brassica juncea (L.) Czern., Carica papaya L., Citrus acida Roxb., C. limon (L.) Burm. etc. are listed in Indian traded medicinal plants list, Abelmoschus moschatus Medik. in CAMP, Psidium guajava L. and Vigna angularis (Willd.) Ohwi \& H. Ohashi in IUCN. Hence, it is urgently required to regroup and clearly demarcate the medicinal plants.

- As many projects have been completed in the past two decades, a database should be prepared at national level, with significant outcomes of the projects. Duplication of work may be avoided and it would help to identify research gap.

- Instead of several projects in parts, a meganetwork project needs to be carried out for ex situ conservation of medicinal plants. As many botanical gardens are being established, but maintaining in botanical gardens/field gene banks is a risky approach, costly affair and more chances of loss of crops due to climate change, pests, diseases and natural calamities. Hence, seed conservation/ in vitro conservation/ cryopreservation may be used for long-term safe conservation.
- Large diversity of any crop is essential for crop improvement, so emphasis may be given that entire genetic diversity of priority medicinal plants are conserved.

- Though India has taken extensive activities to conserve medicinal plants, the collections remain fragmented. There is need for establishment of long-term conservation strategies which are both sustainable and accessible. The ICAR- NBPGR has been conserving all diversity in agri-horticultural crops, where it is mandatory to obtain a national identity- indigenous collection (IC) number before release of any varieties. Similarly for medicinal plants, there is need to follow similar system by all Institutes across India. Recent MOU between ICAR-NBPGR is one such step to enhance focus on conservation of this important group of plants.

- All germplasm with relevant passport information must be conserved in National Gene Bank (seed genebank/in vitro/field genebanks) with a back up duplicate set at regional/Institute level, to not only ensure sustainable availability of diversity for use but also safeguarding threatened medicinal plants for future generations and future unforeseeable diseases/pandemics.

\section{Conclusion}

This paper provides, an easy, updated, ready-to-use guide for information for Indian threatened medicinal plant species, as designated by Indian and global agencies and conservation efforts. Further, online access to data is subject to availability of internet access. Consolidated offline information, as provided in this paper, is often required by students, teachers, policy makers etc., as a ready reckoner. This will help not only the researchers but also policy makers in developing strategies for efficient conservation/cultivation to ensure availability of these precious resources for utilization by future generations.

Acknowledgements Authors are thankful to Director, ICARNBPGR, New Delhi, for encouragement.

Author contributions Gowthami R and Neelam Sharma conceived the idea, gathered information and wrote the draft. Ruchira Pandey and Anuradha Agrawal provided constructive 
feedback and editorial inputs. All authors have reviewed, edited and approved the manuscript.

Funding No funding.

\section{Declaration}

Conflict of interest The authors declare no conflicts of interest.

\section{References}

Ahmadullah M, Nayar MP (1999) Red data book of Indian Plants (Peninsular India): Botanical Survey of India. Calcutta

Anand A (2003) Studies on genetic stability of micropropagated plants and reintroduction in an endemic and endangered taxon: Syzygium travancorium Gamble. J Plant Biotechnol 5:201-207

Bapat VA, Yadav SR, Dixit GB (2008) Rescue of endangered plants through biotechnological applications. Nat Acad Sci Lett 31:201-210

Barik SK, Tiwari ON, Adhikari D, Singh PP, Tiwary R, Barua S (2018) Geographic distribution pattern of threatened plants of India and steps taken for their conservation. Curr Sci 114:470-503

Bhattacharyya R, Bhattacharya S, Chaudhuri S (2006) Conservation and documentation of the medicinal plant resources of India. In: Hawksworth DL, Bull AT (eds) Human Exploitation and Biodiversity Conservation, Springer, Dordrecht, pp 365-377. https://doi.org/10.1007/978-14020-5283-5.

Biswas S, Rawat MS, Tantray FA, Sharma S (2017) Medicinal plants conservation and development areas (MPCDAs) An initiative towards conservation of medicinal plants. Medicinal Plants. Int J Phytomedicines Related Industries 9:143-149. https://doi.org/10.5958/0975-6892.2017. 00022.3

Brooks TM, Bakarr MI, Boucher T, Da Fonseca GA, HiltonTaylor C, Hoekstra JM, Moritz T, Olivieri S, Parrish J, Pressey RL, Rodrigues AS (2004) Coverage provided by the global protected-area system: is it enough? Bioscience 54:1081-1091. https://doi.org/10.1641/0006-3568(2004) 054[1081:CPBTGP]2.0.CO;2

CITES (2017) CITES listed species. https://www.cites.org/eng/ disc/species.php. Accessed on 3.1.2020.

Collar NJ (1996) The reasons for Red Data Books. Oryx 30:121-130. https://doi.org/10.1017/S0030605300021505

Collen B, Dulvy NK, Gaston KJ, Gärdenfors U, Keith DA, Punt AE, Regan HM, Böhm M, Hedges S, Seddon M, Butchart SH (2016) Clarifying misconceptions of extinction risk assessment with the IUCN Red List. Biol Let 12:20150843. https://doi.org/10.1098/rsbl.2015.0843

DBT (2019) Department of Biotechnology. Conservation of Threatened Plants of India. http://www.dbtindia.gov.in/ conservation-threatened-plants-india. Accessed on 4.5.2020.
FRLHTENVIS (2016a) Centre on Medicinal Plants. Medicinal plants under threat. http://envis.frlht.org/overview.html. Accessed on 6.4.2020.

FRLHTENVIS (2016b) Centre on Medicinal Plants. Medicinal Plant Conservation Areas http://envis.frlht.org/mpca.php. Accessed on 6.4.2020.

Goraya GS, Jishtu V, Rawat GS, Ved DK (2013) Wild medicinal plants of Himachal Pradesh: An assessment of their conservation status and management prioritization. Himachal Pradesh Forest Department, Shimla

Goraya GS, Ved DK (2017) Medicinal plants in India: an assessment of their demand and supply. National Medicinal Plants Board, Ministry of AYUSH, Government of India, New Delhi and Indian Council of Forestry Research and Education, Dehradun

Hammer K, Khoshbakht K (2005) Towards a 'red list' for crop plant species. Genet Resour Crop Evol 52:249-265. https:// doi.org/10.1007/s10722-004-7550-6

Hazarika TK, Marak S, Mandal D, Shukla AC (2016) Underutilized and unexploited fruits of Indo-Burma hot spot, Meghalaya, north-east India: ethno-medicinal evaluation, socio-economic importance and conservation strategies. Genet Resour Crop Evol 63:289-304. https:// doi.org/10.1007/s10722-015-0248-0

ICAR-NBPGR (2020) Memorandum of Undertaking signed between National Medicinal Plants Board and ICAR-National Bureau of Plant Genetic Resources on 06-07-2020. http://www.nbpgr.ernet.in/News_Details/aid/249.aspx. Accessed on 23.07.2020.

IUCN (2020) The IUCN red list of threatened species. Version 2020-1. https://www.iucnredlist.org. Accessed on 4.04.2020.

Jadhav SN, Ved DK, Ghate U, Reddy KN, Reddy CS (2001) Proceedings of the workshop on Conservation Assessment and Management Planning for Medicinal Plants of Andhra Pradesh. FRLHT, Bangalore

Jain SK, Rao RR (1983) An assessment of threatened plants of India, Botanical Survey of India, Howrah

Jaisankar I, Velmurugan A, Swarnam TP, Singh AK (2018) Hotspots: an introduction and role in conservation. In: Sivaperuman C, Venkataraman K (eds) Indian Hotspots. Springer, Singapore, pp 1-21. https://doi.org/10.1007/978981-10-6983-3_1.

Kala CP (2000) Status and conservation of rare and endangered medicinal plants of Indian trans-Himalaya. Biol Conserv 93:371-379

Kala CP (2005) Indigenous uses, population density, and conservation of threatened medicinal plants in protected areas of the Indian Himalayas. Conserv Biol 19:368-378. https:// doi.org/10.1111/j.1523-1739.2005.00602.x

Kandari LS, Bisht VK, Bhardwaj M, Thakur AK (2014) Conservation and management of sacred groves, myths and beliefs of tribal communities: a case study from north-India. Environmental Systems Research 3:16. https://doi.org/ 10.1186/s40068-014-0016-8

Krishnan PN, Decruse SW, Radha RK (2011) Conservation of medicinal plants of Western Ghats, India and its sustainable utilization through in vitro technology. Vitro Cell Dev Biol Plant 47:110-122. https://doi.org/10.1007/s11627011-9344-9 
Kumar A, Pandey VC, Singh AG, Tewari DD (2013) Traditional uses of medicinal plants for dermatological healthcare management practices by the Tharu tribal community of Uttar Pradesh, India. Genet Resour Crop Evol 60:203-224. https://doi.org/10.1007/s10722-012-9826-6

KSCSTE-JNTBGRI (2019) Jawaharlal Nehru Tropical Botanic Garden and Research Institute. Herbal garden. https:// jntbgri.res.in/index.php/research/plant-genetic-resources/ medicinal-aromatic-and-spice-plants-unit. Accessed on 15.4.2020.

Kumar G, Srivastava A, Sharma SK, Rao TD, Gupta YK (2015) Efficacy and safety evaluation of Ayurvedic treatment (Ashwagandha powder and Sidh Makardhwaj) in rheumatoid arthritis patients: a pilot prospective study. Indian J Med Res 141:100-106. https://doi.org/10.4103/ 0971-5916.154510

Kumari GP, Joshi C, Tewari LM (2011) Diversity and status of ethno-medicinal plants of Almora district in Uttarakhand India. Int J Biodvers Conserv 3:298-326

Maxted N, Hawkes JG, Guarino L, Sawkins M (1997) Towards the selection of taxa for plant genetic conservation. Genet Resour Crop Evol 44:337-348. https://doi.org/10.1023/A: 1008643206054

Mittermeier RA, Turner WR, Larsen FW, Brooks TM and Gascon C (2011) Global biodiversity conservation: the critical role of hotspots. In Zachos F, Habel J (eds) Biodiversity hotspots. Springer, Berlin, Heidelberg, pp. 3-22. https://doi.org/10.1007/978-3-642-20992-5_1.

Myers N, Mittermeier RA, Mittermeier CG, Da Fonseca GA, Kent J (2000) Biodiversity hotspots for conservation priorities. Nature 403:853-858. https://doi.org/10.1038/ 35002501

Nayar MP and Sastry ARK (1987-1990) Red Data Book of Indian Plants, Botanical Survey of India, Calcutta, vols $3: 15$.

NBA (2020) National Biodiversity Authority. Species of Plants and animals which are on the verge of extinction in different states of India. http://nbaindia.org/content/500/55/1/ biodiversityrelatedi.html. Accessed on 25.01.2020.

NMPB (2020a) Demand and supply of medicinal plants. National Medicinal Plants Board (NMPB). https://nmpb. nic.in/medicinal_list. Accessed on 27.07.2020.

NMPB (2020b) National Medicinal Plants Board (NMPB). https://www.nmpb.nic.in/ (accessed on 31.1.2020).

Pareek SK, Gupta V, Bhatt KC, Negi KS, Sharma N (2005) Medicinal and aromatic plants. In: Dhillon BS, Tyagi RK, Saxena S, Randhawa GJ (eds) Plant genetic resources: horticultural crops. Narosa Publishing House, New Delhi, pp 279-308

Patwardhan B, Chavan-Gautam P, Gautam M, Tillu G, Chopra A, Gairola S, Jadhav S (2020) Ayurveda rasayana in prophylaxis of covid-19. Curr Sci 118:1158-1160

Pelletier TA, Carstens BC, Tank DC, Sullivan J, Espíndola A (2018) Predicting plant conservation priorities on a global scale. Proc Natl Acad Sci USA 115:13027-13032. https:// doi.org/10.1073/pnas.1804098115

Pollock C, Mace GM, Hilton-Taylor C (2003) The revised IUCN Red List Categories and Criteria, Version 3.1. In: de Iongh HH, Bánki OS, Bergmans W, van der Werff ten Bosch, MJ (eds) The Harmonization of Red Lists for Threatened Species in Europe. Leiden (The Netherlands):
The Netherlands Commission for International Nature Protection, pp 33-48.

Rajpurohit D, Jhang T (2015) In situ and ex situ conservation of plant genetic resources and traditional knowledge. In: Salgotra RK, Gupta BB (eds) Plant Genetic Resources and Traditional Knowledge for Food Security. Springer, Berlin, pp 137-162

Rao CK, Geetha BL, Suresh G (2003) Red List of Threatened Vascular Plant Species in India, Botanical Survey of India, Howrah, pp ix-144.

Ravikanth G, Jagadish MR, Vasudeva R, Shaanker RU, Aravind NA (2018) Recovery of critically endangered plant species in India: need for a comprehensive approach. Curr Sci 114:504

Rodrigues AS, Pilgrim JD, Lamoreux JF, Hoffmann M, Brooks TM (2006) The value of the IUCN Red List for conservation. Trends Ecol Evol 21:71-76. https://doi.org/10. 1016/j.tree.2005.10.010

Sarker SD, Nahar L (2007) Chemistry for Pharmacy Students General, Organic and Natural Product Chemistry. Wiley, England, pp 283-359

Seeni S, Latha PG (2000) In vitro multiplication and eco-rehabilitation of the endangered Blue Vanda. Plant Cell Tissue Organ Cult 61:1-8. https://doi.org/10.1023/A: 1006444614657

Shakya AK (2016) Medicinal plants: future source of new drugs. Int J Herb Med 4:59-64

Sharma N, Gowthami R, Pandey R (2019) Synthetic Seeds: A Valuable Adjunct for Conservation of Medicinal Plants. In: Faisal M, Alatar AA (eds) Synthetic Seeds - Germplasm Regeneration, Preservation and Prospects. Springer Nature, Switzerland, pp 181-216. doi.org/https://doi.org/10. 1007/978-3-030-24631-0_7.

Sharma N, Pandey R (2013) Conservation of medicinal plants in Tropics. In: Normah MN, Chin HF, Reed BM (eds), Conservation of tropical plant species. Springer, New York, pp 437-487. https://doi.org/10.1007/978-1-4614-3776-5.

Sharma N, Pandey R, Gowthami R (2020) In vitro conservation and cryopreservation of threatened medicinal plants of India. In: Rajasekharan PE, Wani SH (eds) Conservation and utilization of threatened medicinal plants. Springer, India, pp 181-228. https://doi.org/10.1007/978-3-03039793-7

Shivanna KR (2020) The Sixth Mass Extinction Crisis and its Impact on Biodiversity and Human Welfare. Resonance 25:93-109. https://doi.org/10.1007/s12045-019-0924-z

Singh JP, Kumar S, Venkatesan K, Kulloli RN (2016) Conservation status and utilization of Caralluma edulis: an important threatened medicinal plant species of the Thar Desert, India. Genet Resour Crop Evol 63:721-732. https:// doi.org/10.1007/s10722-016-0366-3

Singh N, Pandey S (2019) Conservation of plant genetic resources. In: Pandey CD, Koul AK, Vimala Devi S, Singh N, Radhamani J, Pandey S, Jacob SR, Aravind J, Gore PG, Gupta V (eds) International training programme on management of plant genetic resources for officers from directorate of seed testing and certification ministry of agriculture, Baghdad. Republic of Iraq, ICAR-NBPGR, New Delhi, p 27 
Ved DK, Tandon V (1998) CAMP report for high altitude medicinal plants of Jammu-Kashmir and Himachal Pradesh. FRLHT, Bangalore, India

Ved DK, Goraya GS (2008) Demand and supply of medicinal plants in India. Bishen Singh, Mahendra Pal Singh, Dehradun and FRLHT, Bangalore, India

Ved DK, Kinhal GA, Haridasan K, Ravikumar K, Ghate U, Shankar RV, Indresha J H (2003a) Conservation assessment and management prioritization for the medicinal plants of Arunachal Pradesh, Assam, Meghalaya and Sikkim- Proceedings of the workshop held at Guwahati during $27^{\text {th }}$ February to 1 st March 2003. FRLHT, Bangalore

Ved DK, Kinhal GK, Ravikumar K, Prabhakaran V, Ghate U, Sankar RV, Indresha JH (2003b) Conservation assessment management prioritization for the Medicinal plants of Jammu and Kashmir, Himachal Pradesh and Uttaranchal. FRLHT, Bangalore

Ved DK, Kinhal GA, Ravikumar K, Sankar RV, Haridasan K (2005) Conservation Assessment and Management Prioritisation (CAMP) for wild medicinal plants of North-East India. Medicinal Plant Conservation 11:40-44

Ved DK, Suma TS, Barve V, Srinivas V, Sangeetha S, Ravikumar K, Kartikeyan R, Kulkarni V, Kumar AS, Venugopal SN, Somashekhar BS, Sumanth MV, Begum N, Sugandhi Rani, Surekha KV, Desale N (2016a) State wise CAMP workshop details. (envis.frlht.org/frlhtenvis.nic.in) (http://envis.frlht.org/conservation-concern-species). FRLHT's ENVIS Centre on Medicinal Plants, Bengaluru. Copy Right: FRLHT, Bengaluru and MoEFCC, GoI. Accessed on 6.4.2020.

Ved DK, Suma TS, Barve V, Srinivas V, Sangeetha S, Ravikumar K, Kartikeyan R, Kulkarni V, Kumar AS, Venugopal SN, Somashekhar BS, Sumanth MV, Begum N, Sugandhi Rani, Surekha KV, Desale N (2016b) State wise list of medicinal plants of conservation concern. (envis.frlht.org/frlhtenvis.nic.in) (http://envis.frlht.org/mpccspecies). FRLHT's ENVIS Centre on Medicinal Plants, Bengaluru. Copy Right: FRLHT, Bengaluru and MoEFCC, GoI. Accessed on 6.4.2020.

Walter KS, Gillett HJ (1998) 1997 IUCN Red list of threatened plants. Compiled by the World Conservation Monitoring Centre, IUCN-The World Conservation Union, Gland, Switzerland and Cambridge, UK.

WIIENVIS (2019) ENVIS Centre on Wildlife and Protected Areas. Protected Areas of India. http://wiienvis.nic.in/ Database/Protected_Area_854.aspx. Accessed on 20.2.2020.

Publisher's Note Springer Nature remains neutral with regard to jurisdictional claims in published maps and institutional affiliations. 